University of Nebraska - Lincoln

DigitalCommons@University of Nebraska - Lincoln

2001

Designing Adaptive Low-Dissipative High Order Schemes for

Long-Time Integrations

Helen Yee

Bjorn Sjögreen

Follow this and additional works at: https://digitalcommons.unl.edu/nasapub

Part of the Astrophysics and Astronomy Commons

This Article is brought to you for free and open access by the National Aeronautics and Space Administration at DigitalCommons@University of Nebraska - Lincoln. It has been accepted for inclusion in NASA Publications by an authorized administrator of DigitalCommons@University of Nebraska - Lincoln. 


\title{
DESIGNING ADAPTIVE LOW-DISSIPATIVE HIGH ORDER SCHEMES FOR LONG-TIME INTEGRATIONS*
}

\author{
H. C. Yee \\ NASA Ames Research Center, Moffett Field,CA 94035, USA \\ yee@nas.nasa.gov \\ B. Sjögreen \\ Department of Numerical Analysis and Computer Sciences, \\ KTH, 10044 Stockholm, Sweden. \\ bjorns@nada.kth.se
}

\begin{abstract}
A general framework for the design of adaptive low-dissipative high order schemes is presented. It encompasses a rather complete treatment of the numerical approach based on four integrated design criteria: (1) For stability considerations, condition the governing equations before the application of the appropriate numerical scheme whenever it is possible. (2) For consistency, compatible schemes that possess stability properties, including physical and numerical boundary condition treatments, similar to those of the discrete analogue of the continuum are preferred. (3) For the minimization of numerical dissipation contamination, efficient and adaptive numerical dissipation control to further improve nonlinear stability and accuracy should be used. (4) For practical considerations, the numerical approach should be efficient and applicable to general geometries, and an efficient and reliable dynamic grid adaptation should be used if necessary. These design criteria are, in general, very useful to a wide spectrum of flow simulations. However, the demand on the overall numerical approach for nonlinear stability and accuracy is much more stringent for long-time integration of complex multiscale viscous shock/shear/turbulence/acoustics interactions and numerical combustion. Robust classical numerical methods for less complex flow physics are not suitable or practical for such applications. The present approach is designed expressly to address such flow problems, especially unsteady flows. The minimization of employing very fine grids to overcome the production
\end{abstract}

\footnotetext{
*A chapter for Turbulent Flow Computation, (eds. D. Drikakis \& B. Geurts), Kluwer Academic Publisher, 2001. Expanded version of the paper for the Proceedings of the Third AFOSR International Conference on DNS/LES, Arlington Texas, August 4-9, 2001. Part of this work was carried out while the second author was a visiting scientist with RIACS, NASA Ames Research Center.
} 
of spurious numerical solutions and/or instability due to under-resolved grids is also sought $[79,17]$. The incremental studies to illustrate the performance of the approach are summarized. Extensive testing and full implementation of the approach is forthcoming. The results shown so far are very encouraging.

Keywords: Low-Dissipative Schemes, Adaptive Numerical Dissipation/Filer Controls, High Order Finite Difference Methods, Linear and Nonlinear Instabilities, Skew-Symmetric Form, Entropy Splitting, Summation-by-Parts, Integration-by-Parts, Wavelets, Multi-Resolution Wavelets, linear and nonlinear filters.

\section{Introduction}

Classical stability and convergence theory are based on linear and local linearized analysis as the time steps and grid spacings approach zero. This theory offers no guarantee for nonlinear stability and convergence to the correct solution of the nonlinear governing equations. The use of numerical dissipation has been the key mechanism in combating numerical instabilities. Recent nonlinear stability analysis based on energy norm estimate [24] offers stability improvement in combating nonlinear instability for long-time integrations and/or simple smooth flows. These recent developments offer two major sources of stabilizing mechanisms, namely, from the governing equation level and from the numerical scheme level. Employing a nonlinear stable form of the governing equation in conjunction with appropriate nonlinear schemes for initial-boundary-value problems (IBVPs) is one way of minimizing the use of numerical dissipations $[56,55]$. On the other hand, even with the recent development, when employing finite time steps and finite grid spacings in the long-time integration of multiscale complex nonlinear fluid flows, nonlinear instability, although greatly reduced, still occurs and the use of a numerical-dissipation/filter is unavoidable.

Aside from acting as a post-processor step, most filters serve as some form of numerical dissipation. Without loss of generality, "numerical-dissipation/filter" is, hereafter, referred to as "numerical dissipation" unless otherwise stated. Proper control of the numerical dissipation to accurately resolve all relevant multiscales of complex flow problems while still maintaining nonlinear stability and efficiency for long-time numerical integrations poses a great challenge to the design of numerical methods. The required type and amount of numerical dissipation are not only physical problem dependent, but also vary from one flow region to another. This is particularly true for unsteady high-speed shock/shear/boundary-layer/turbulence/acoustics interactions and/or combustion problems, since the dynamics of the nonlinear effect of these flows are not well-understood [79], while long-time integrations of these flows have already stretched the limit of the currently available supercomputers and the existing numerical methods. It is of paramount importance to have proper control of the type and amount of numerical dissipation in regions where it is needed 
but nowhere else. Inappropriate type and/or amount can be detrimental to the integrity of the computed solution even with extensive grid refinement.

The present work is a sequel to $[82,83,63,65]$. It is an expanded version of [84]. The objective here is to propose a rather complete treatment of the numerical approach based on the four integrated design criteria (1)-(4) stated in the abstract. The key emphasis here is to describe and illustrate with examples on an adaptive procedure employing appropriate sensors to switch on the desired numerical dissipation where needed, and leave the rest of the region free of numerical dissipation contamination, while at the same time improving nonlinear stability of the entire numerical process for long-time numerical integration of the complex multiscale problems in question. These sensors are capable of distinguishing shocks/shears from turbulent fluctuations and/or spurious highfrequency oscillations for a full spectrum of flow speeds and Reynolds numbers. The minimization of employing very fine grids to overcome the production of spurious numerical solutions and/or instability due to under-resolved grids is sought [17]. It was shown in $[56,20,83,63]$ that conditioning the governing equations via the so called entropy splitting of the inviscid flux derivatives [83] can improve the over all stability of the numerical approach for smooth flows. Therefore, the same shock/shear detector that is designed to switch on the shock/shear numerical dissipation can be used to switch off the entropy splitting form of the inviscid flux derivative in the vicinity the discontinuous regions to further improve nonlinear stability and minimize the use of numerical dissipation. The rest of the sensors, in conjunction with the local flow speed and Reynolds number, can also be used to adaptively determine the appropriate entropy splitting parameter for each flow type/region. These sensors are readily available as an improvement over existing grid adaptation indicators [20]. If applied correctly, the proposed adaptive numerical dissipation control is scheme independent, and can be a stand alone option for many of the favorite schemes used in the literature.

Outline: A brief summary of linear and nonlinear stability and the logistics of advocating design criteria (1)-(4) for a complete numerical approach are discussed in Sections $2-4$. Adaptive numerical dissipation controls for high order schemes are discussed in Section 5. Some representative examples to illustrate the performance of the approach are given in Section 6.

\section{Conditioning of the Governing Equations}

Traditionally, conditioning the governing partial differential equations (PDEs) usually referred to rewriting the governing equations in an equivalent set of PDEs in order to prove the stability and/or well-posedness of the PDEs. When numerical methods are used to solve PDEs that are nonlinear, it is well-known that different equivalent forms of the governing equations might exhibit dif- 
ferent numerical stability, accuracy and/or spurious computed solutions, even for problems containing no shock/shear discontinuities. There are many conditioned forms of the governing equations proposed in the literature. Different conditioned forms of the nonlinear convection fluxes and the viscous fluxes have been proposed for the incompressible and compressible Navier-Stokes equations. Here we concentrate on the convection terms of these equations and mention a few conditioning forms which are precursors of the so called entropy splitting of the compressible Euler equations [83]. If a method-of-lines approach is used to discretize these equations, the entropy splitting reduces to the splitting of the convection flux derivatives. For the viscous terms, we only adapt the method of preventing odd and even decoupling on all of our numerical experiments whenever it is applicable [61].

The splitting of the nonlinear convection terms (for both the compressible and incompressible Navier-Stokes equations) into a conservative part and a non-conservative part has been known for a long time. In the DNS, LES and atmospheric science simulation literature, it is referred to as the skewsymmetric form of the momentum equations $[4,30,5,86]$. It consists of the mean average of the conservative and non-conservative (convective form [86]) part of the momentum equations. The spatial difference operator is then applied to the split form. From the numerical analysis standpoint, the Hirt and Zalesak's ZIP scheme [27, 85] is equivalent to applying central schemes to the non-conservative momentum equations (convective form of the momentum equations). MacCormack [39] proposed the use of the skew-symmetric form for problems other than DNS and LES. Harten [25] and Tadmor [73] discussed the symmetric form of the Euler equations and skew-adjoint form of hyperbolic conservation laws, respectively. Although the derivation in these works is different, the ultimate goal of using the split form is almost identical. This goal is to improve nonlinear stability, minimize spurious high-frequency oscillations, and enhance robustness of the numerical computations. The canonical splitting used by Olsson \& Oliger [56] is a mathematical tool to prove the existence of a generalized energy estimate for a symmetrizable system of conservation laws. For the thermally perfect gas compressible Euler equations, the transformation consists of a convex entropy function that satisfies a mathematical entropy condition. The mathematical entropy function, in this case, can be a function of the physical entropy. Therefore, the resulting splitting was referred to as entropy splitting by Yee et al. [83]. The entropy splitting can be viewed as a more general form than its precursors which makes possible the $L^{2}$ stability proof of the nonlinear Euler equations with physical boundary conditions (BCs) included. The following subsections which were part of [65], provide more details. 


\subsection{Introduction to skew-symmetric splitting}

Consider a variable coefficient linear hyperbolic system

$$
U_{t}+A(x) U_{x}=0 \quad a<x<b,
$$

where $U$ is a vector and the matrix $A(x)$ is symmetric. Define the scalar product and norm,

$$
(U, V)=\int_{a}^{b} U(x)^{T} V(x) d x, \quad\|U\|^{2}=(U, U)
$$

It is possible to obtain an energy estimate for the solution by integration-byparts. To do this, write the system in skew-symmetric form,

$$
U_{t}+\frac{1}{2}(A(x) U)_{x}+\frac{1}{2} A(x) U_{x}-\frac{1}{2} A_{x} U=0 .
$$

Start from

$$
\frac{1}{2} \frac{d}{d t}\|U\|^{2}=\left(U, U_{t}\right)=-\frac{1}{2}\left[\left(U,(A(x) U)_{x}\right)+\left(U, A(x) U_{x}\right)+\left(U, A_{x} U\right)\right]
$$

and perform the integration-by-parts

$$
\left(U,(A(x) U)_{x}\right)=-\left(U_{x}, A(x) U\right)+\left[U^{T} A U\right]_{a}^{b}=-\left(U, A U_{x}\right)+\left[U^{T} A U\right]_{a}^{b}
$$

where the last equality follows from the symmetry of $A$. This gives the energy norm estimate

$$
\frac{1}{2} \frac{d}{d t}\|U\|^{2}=-\frac{1}{2}\left(\left[U^{T} A U\right]_{a}^{b}-\left(A_{x} U, U\right)\right),
$$

which is a standard result that has been known for a long time. It can be found in many textbooks on PDEs, e.g., [19].

For semi-discrete difference approximations, the same idea can be used. Introduce the grid points $x_{j}=a+(j-1) h, j=1,2, \ldots, N$ on the interval $[a, b]$, with uniform grid spacing $h=(b-a) /(N-1)$. Apply a spatial discretization to the skew-symmetric form

$$
\frac{d U_{j}(t)}{d t}=-\frac{1}{2} A\left(x_{j}\right) D U_{j}-\frac{1}{2} D\left(A\left(x_{j}\right) U_{j}\right)+\frac{1}{2} D A\left(x_{j}\right) U_{j}
$$

where $D$ is a finite difference operator, approximating the spatial derivative. We will obtain an estimate in a discrete scalar product,

$$
(U, V)_{h}=h \sum_{i, j=1}^{N} \sigma_{i, j} U_{i} V_{j}
$$


in the same way as for the continuous case. Here $\sigma_{i, j}$ a positive definite matrix (identity matrix for the $L^{2}$ norm). The estimate becomes

$$
\begin{aligned}
\frac{1}{2} \frac{d}{d t}\|U\|_{h}^{2}= & -\frac{1}{2}\left((U, A D U)_{h}+(U, D(A U))_{h}\right)+\frac{1}{2}(U, D(A) U)_{h}= \\
& -\frac{1}{2}\left(U_{N}^{T} A_{N} U_{N}-U_{1}^{T} A_{1} U_{1}\right)+\frac{1}{2}(U, D(A) U)_{h},
\end{aligned}
$$

where we now assume that the difference operator has the summation-by-parts (SBP) property,

$$
(U, D V)_{h}=-(D U, V)_{h}+U_{N} V_{N}-U_{1} V_{1}
$$

with respect to the discrete scalar product. The SBP property here is the discrete analogue of the integration-by-parts energy norm property. One simple SBP operator is given by

$$
\begin{aligned}
& D U_{1}=D_{+} U_{1} \\
& D U_{j}=D_{0} U_{j}, \quad j=2,3, \ldots, N-1 \\
& D U_{N}=D_{-} U_{N}
\end{aligned}
$$

for the scalar product

$$
(U, V)_{h}=\frac{h}{2} U_{1} V_{1}+h \sum_{j=2}^{N-1} U_{j} V_{j}+\frac{h}{2} U_{N} V_{N} .
$$

Here we define $D_{0} U_{j}=\left(U_{j+1}-U_{j-1}\right) /(2 h), D_{+}=\left(U_{j+1}-U_{j}\right) / h, D_{-} U_{j}=$ $\left(U_{j}-U_{j-1}\right) / h$. Higher order accurate SBP operators can be found; see [70]. For periodic problems, the SBP property is usually easy to verify. In this case the boundary terms disappear.

The crucial point is the splitting of the convective term,

$$
A U_{x}=\frac{1}{2} A U_{x}+\frac{1}{2}(A U)_{x}-\frac{1}{2} A_{x} U,
$$

into one conservative and one non-conservative parts. The difference approximation is applied to the split form. The skew-symmetric splitting for difference approximations has also been known for a long time. It was used in [30], and [31] to prove estimates for the Fourier method. See also [47], where SBP is proved for the Fourier method and a fourth-order difference method, when the boundaries are periodic.

Although this $L^{2}$ estimate does not give uniform boundedness of the solution, it has turned out in practical computations that methods based on skewsymmetric splitting perform much better for long-time integrations than un-split methods. 
Actually, for a symmetric hyperbolic nonlinear system with $A(x)$ replaced by $A(U(x))$, similar skew-symmetric splitting and energy norm can be obtained. One of the earliest works on skew-symmetric splitting is Arakawa [4], where a splitting was derived for the 2D Euler equations for incompressible fluid flow in vorticity stream function formulation,

$$
\omega_{t}=\psi_{y} \omega_{x}-\psi_{x} \omega_{y}
$$

Here $\omega$ is the vorticity and $\psi$ is the stream function, such that the velocity $(u, v)$ is $\left(-\psi_{y}, \psi_{x}\right)$. In [4] it is shown that the approximation

$$
\begin{aligned}
\frac{d \omega_{i, j}(t)}{d t}= & \frac{1}{3}\left(D_{y} \psi_{i, j} D_{x} \omega_{i, j}-D_{x} \psi_{i, j} D_{y} \omega_{i, j}\right)+ \\
& \frac{1}{3}\left(D_{x}\left(\omega_{i, j} D_{y} \psi_{i, j}\right)-D_{y}\left(\omega_{i, j} D_{x} \psi_{i, j}\right)\right)+ \\
& \frac{1}{3}\left(D_{y}\left(\psi_{i, j} D_{x} \omega_{i, j}\right)-D_{x}\left(\psi_{i, j} D_{y} \omega_{i, j}\right)\right)
\end{aligned}
$$

leads to the estimates

$$
\begin{aligned}
& \frac{d}{d t}\|\omega\|^{2}=\left(\omega, \omega_{t}\right)_{h}=0 \\
& \left(\psi, \omega_{t}\right)_{h}=0 .
\end{aligned}
$$

Here it is assumed that boundary terms are equal to zero (homogeneous). $D_{x}$ and $D_{y}$ denote finite difference operators acting in the $x$ - and $y$-direction respectively. The second estimate is related to the conservation of kinetic energy,

$$
\frac{1}{2} \frac{d}{d t}\left(\left\|\psi_{y}\right\|^{2}+\left\|\psi_{x}\right\|^{2}\right)=-\left(\psi, \omega_{t}\right)
$$

The proof of the estimates only involves pairwise cancellation of terms according to the rule,

$$
(u, D(u v))_{h}+(u, v D u)_{h}=0,
$$

which holds for zero boundary data, if $D$ satisfies (2.10). In [4], the operator (2.11) is used. Note that the use of $\omega$ and $\psi$ in this section pertains to the vorticicity formulation symbols. In later sections, the same symbols will have different meanings.

In [30], the inviscid Burgers' equation,

$$
u_{t}+\left(u^{2} / 2\right)_{x}=0
$$

with the quadratic flux derivative split into $\frac{1}{3} u u_{x}+\frac{2}{3}\left(u^{2} / 2\right) x$, is approximated as

$$
\frac{d u_{j}}{d t}=-\frac{1}{3}\left(u_{j} D u_{j}+D u_{j}^{2}\right)
$$


For this approximation, we obtain

$$
\frac{1}{2} \frac{d}{d t}\|u\|_{h}^{2}=-\frac{1}{3}\left((u, u D u)_{h}+\left(u, D u^{2}\right)_{h}\right)=0
$$

by using SBP on the last term. Again, boundary data is assumed to be zero. The split form $\left(D u^{2}+u D u\right) / 3$ is also used in [58].

It is important to note that the split form is non-dissipative in the sense that the highest grid frequency, $u_{j}=(-1)^{j}$, has derivative zero, and thus cannot be seen or smoothed by the time integration. In addition, the difference operator applied to the split form should be done with $D$ on the non-conservative term, and $-D^{T}$, the negative adjoint of $D$, on the conservative term. However, since even order centered difference operators are anti-symmetric, we write $D$ in both places. For odd orders of accuracy, the approximation should be done analogously to the first order example,

$$
\frac{d u_{j}}{d t}=-\frac{1}{3}\left(u_{j} D_{+} u_{j}+D_{-} u_{j}^{2}\right) .
$$

\subsection{Skew-Symmetric Splitting for Incompressible Fluid Flow}

For a 3-D incompressible Navier-Stokes equations of the form,

$$
\begin{aligned}
& \mathbf{u}_{t}+\left(\mathbf{u}^{T} \nabla\right) \mathbf{u}=-\nabla p+\nu \triangle \mathbf{u}, \\
& \operatorname{div} \mathbf{u}=0
\end{aligned}
$$

skew-symmetric splitting can be applied on the convective terms to estimate the kinetic energy, $\mathbf{u}^{T} \mathbf{u}$. Here the velocity vector is $\mathbf{u}=\left(u_{1}, u_{2}, u_{3}\right)^{T}$, the pressure $p$ and the viscosity coefficient is $\nu$. In [86] and in [26] the three forms

$$
\begin{array}{ll}
\left(\mathbf{u}^{T} \nabla\right) \mathbf{u} & \text { (convective) } \\
\frac{1}{2}\left(\left(\mathbf{u}^{T} \nabla\right) \mathbf{u}+\operatorname{div}\left(\mathbf{u u}^{T}\right)\right) & \text { (skew-symmetric) } \\
\frac{1}{2}\left(\nabla\left(\mathbf{u}^{T} \mathbf{u}\right)+\mathbf{u} \times \nabla \times \mathbf{u}\right) & \text { (rotational) }
\end{array}
$$

for the nonlinear terms are studied. They are equivalent to each other before the application of the numerical methods. Although the skew-symmetric and rotational forms are not in conservative form, they lead to conservation of kinetic energy that is important for long-time integration. For the inviscid case $\nu=0$, when using the skew-symmetric form, we can estimate the kinetic energy as,

$$
\begin{aligned}
\frac{1}{2} \frac{d}{d t}\left(\left\|\sqrt{\mathbf{u}^{T} \mathbf{u}}\right\|^{2}\right) & =\left(u_{1},\left(u_{1}\right)_{t}\right)+\left(u_{2},\left(u_{2}\right)_{t}\right)+\left(u_{3},\left(u_{3}\right)_{t}\right)= \\
& -\left(u_{i}, u_{j} \partial_{j} u_{i}\right)-\left(u_{i}, \partial_{j}\left(u_{i} u_{j}\right)\right)-\left(u_{i}, \partial_{i} p\right)= \\
& -\left(u_{i}, u_{j} \partial_{j} u_{i}\right)+\left(\partial_{j} u_{i}, u_{i} u_{j}\right)+\left(\partial_{i} u_{i}, p\right)=0
\end{aligned}
$$


where the summation convention is used. We assume that boundary velocities are zero. Integration-by-parts used above shows that the three pressure components are equal to $(\operatorname{div}(\mathbf{u}), p)$, which is zero since the divergence is zero.

In order to carry out the same estimate for a difference approximation, we should use the skew-symmetric form for the convective terms, and discretize by a difference operator having the SBP property. The convective terms then disappear from the estimate directly, without use of the divergence condition, just as for the PDE. To eliminate the pressure term, it is enough that the pressure derivatives and the divergence condition are discretized by the same difference operator (or by operators that are negative adjoints, in the case of odd order of accuracy). We end up with

$$
\frac{1}{2} \frac{d}{d t}\left\|\mathbf{u}_{i, j, k}^{T} \mathbf{u}_{i, j, k}\right\|_{h}^{2} \leq 0
$$

for the difference approximation in a discrete norm. Note that the inequality (2.26) should really be an equality in the setting it is proved. Perhaps it is possible to keep the viscous terms, and make it an inequality.

The discrete estimate can also be derived from the discretized rotational form (as in [47]), but then the discretized divergence condition must be used to eliminate certain convective terms. For this to be possible, it is necessary that the divergence condition is discretized by the same operator as used for the other convection terms. Results from using the skew-symmetric form are compared with results from the rotational form in a turbulence simulation in [86]. The skew-symmetric form is found to give more accurate results. It is recommended that the pressure equation and the divergence condition should be discretized by the same SBP satisfying operator (or SBP operator for ease of reference), so that we can eliminate the term $\left(u_{1}, p_{x}\right)+\left(u_{2}, p_{y}\right)+\left(u_{3}, p_{z}\right)$.

\subsection{Skew-Symmetric Splitting for Compressible Fluid Flows}

Consider the equations of inviscid compressible fluid flow in one space dimension

$$
\left(\begin{array}{c}
\rho \\
\rho u \\
e
\end{array}\right)_{t}+\left(\begin{array}{c}
\rho u \\
\rho u^{2}+p \\
u(e+p)
\end{array}\right)_{x}=\left(\begin{array}{l}
0 \\
0 \\
0
\end{array}\right)
$$

with $\rho, u, e$ and $p$, the density, velocity, total energy per unit volume and pressure, respectively. In [5], a skew-symmetric splitting of the convective terms in momentum equation is used. This splitting was originally presented in [16]. The discretization in [5] is made for a more general equation, but for 
the simple equation (2.27), it becomes

$$
\frac{d}{d t}\left(\begin{array}{c}
\rho_{j}(t) \\
(\rho u)_{j}(t) \\
e_{j}(t)
\end{array}\right)+\left(\begin{array}{c}
D\left(\rho_{j} u_{j}\right) \\
\frac{1}{2} D\left(\rho_{j} u_{j}^{2}\right)+\frac{1}{2} \rho_{j} u_{j} D u_{j}+\frac{1}{2} u_{j} D\left(\rho_{j} u_{j}\right)+D p_{j} \\
D\left(u_{j}\left(e_{j}+p_{j}\right)\right)
\end{array}\right)=\left(\begin{array}{l}
0 \\
0 \\
0
\end{array}\right),
$$

where we now only consider semi-discrete approximations. The skew-symmetric splitting of the convective term in the momentum equation makes it possible to estimate

$$
\frac{d}{d t} h \sum_{j=1}^{N} \rho_{j} u_{j}^{2}=-h \sum_{j=1}^{N} u_{j} D p_{j}+\text { boundary terms }
$$

which is the discrete analogue of the estimate,

$$
\frac{d}{d t}\left\|\rho u^{2}\right\|^{2}=-\left(u, p_{x}\right)+\text { boundary terms }
$$

obtained from the PDE.

\subsection{Canonical (Entropy) Splitting for Systems of Conservation Laws}

The skew-symmetric splitting for the nonlinear incompressible and compressible Navier-Stokes equations discussed above only involve the nonlinear convective terms of the momentum equation, and not the entire inviscid flux derivatives of the PDEs. Actually, for a general nonlinear system of conservation laws,

$$
U_{t}+F(U)_{x}=0, \quad a<x<b, \quad 0<t,
$$

we can perform a skew-symmetric splitting of the entire inviscid flux vector derivative $F(U)_{x}$, if

$1 A(U)=\partial F / \partial U$ is symmetric.

$2 F$ is homogeneous, i.e., $F(\lambda U)=\lambda^{\beta} F(U)$, with $\beta \neq-1$.

It is possible to show that

$$
A(U) U=\beta F(U),
$$

by differentiating the homogeneity relation with respect to $\lambda$, and setting $\lambda=1$. Define the splitting as

$$
U_{t}+\frac{1}{1+\beta} A(U) U_{x}+\frac{\beta}{1+\beta} F(U)_{x}=0 .
$$


We then can show that

$$
\begin{aligned}
\frac{1}{2} \frac{d}{d t} & \|U\|^{2}=\left(U, U_{t}\right)=-\frac{1}{1+\beta}\left(U, A(U) U_{x}\right)-\frac{\beta}{1+\beta}\left(U, F(U)_{x}\right)= \\
& -\frac{1}{1+\beta}\left(U, A(U) U_{x}\right)+\frac{\beta}{1+\beta}\left(U_{x}, F(U)\right)-\frac{\beta}{1+\beta}\left[U^{T} F(U)\right]_{a}^{b}= \\
& -\frac{1}{1+\beta}\left[U^{T} A U\right]_{a}^{b} .
\end{aligned}
$$

The two inner products disappear due to (2.32), and we are left with an estimate of the norm of the solution in terms of the solution on the boundary, a so called generalized energy estimate [56]. Olsson \& Oliger [56] used the term generalized energy estimate because the norm will, in general, depend on an entropy vector $W$, but not the gradient of $U$ in symmetrizable conservation laws.

In [56] the splitting is first done without the homogeneity assumptions. In that case, the entropy flux function $F^{E}(U)$

$$
F^{E}(U)=\int_{0}^{1} F(\theta U) d \theta
$$

is introduced. It follows that

$$
F_{U}^{E}(U) U=-F^{E}+F
$$

so that

$$
F_{x}=\left(F_{U}^{E} U\right)_{x}+F_{U}^{E} U_{x}
$$

If $F_{U}$ is symmetric, then $F_{U}^{E}$ is symmetric too, and we obtain,

$$
\begin{aligned}
\left(U, F_{x}\right) & =\left(U,\left(F_{U}^{E} U\right)_{x}\right)+\left(U, F_{U}^{E} U_{x}\right)= \\
& -\left(U_{x}, F_{U}^{E} U\right)+\left(U, F_{U}^{E} U_{x}\right)+\left[U F_{U}^{E} U\right]_{a}^{b}=\left[U^{T} F_{U}^{E} U\right]_{a}^{b}
\end{aligned}
$$

If the flux function $F$ is homogeneous, $F^{E}$ is just a scalar times $F$, and we recover the splitting (2.33).

In practice, the Jacobian matrix $A(U)$ is not symmetric, especially for more than 1-D. However, in many cases a symmetrizing variable transformation is available. The estimate for the homogeneous case above and symmetrizing transformations were given in [73]. In [56] the analysis is extended to nonhomogeneous problems, and BCs are discussed in greater detail using the so called canonical splitting of the symmetrizable conservation laws. Formulas for symmetrizing the nonlinear compressible Euler equations are given in [25], and the corresponding analytical form for the canonical splitting of the perfect gas compressible case is given in [20]. It was further extended to thermally perfect gases and to 3-D generalized coordinates that preserve freestream in $[83,77]$. 
Let the symmetrizing vector $W$ be related to $U$ via a transformation,

$$
U=U(W)
$$

It can be proved that the existence of a symmetrizing transformation is equivalent to the existence of an entropy function, $E(U)$, with $E_{U U}$ positive definite. The entropy function is a function such that

$$
E_{t}+F_{x}^{E}=0
$$

is an additional conservation law, obtained by multiplying the original conservation law by $E_{U}(U) . F^{E}$ is the entropy flux, related to the entropy by

$$
\left(F_{U}^{E}\right)^{T}=E_{U}^{T} F_{U}
$$

an equation which is overdetermined, and therefore does not have a solution for all systems of conservation laws. Written in terms of the entropy function, (the inverse of) the symmetrizing change of variables (2.39) is defined by $W=$ $E_{U}(U)$. The change of variables $\partial U / \partial W$ is symmetric and positive definite, and the new Jacobian $\partial F / \partial W$ is symmetric. If furthermore $U$ and $F$ are homogeneous in $W$ of degree $\beta$, which is the case for the thermally perfect gas Euler equations for any $\beta \neq-1$, the formulas become simple. In that case we insert the change of variables into the conservation law and obtain

$$
U_{W} W_{t}+F_{W} W_{x}=0
$$

and define the split form of the flux derivative [56]

$$
U_{t}+\frac{\beta}{1+\beta} F_{x}+\frac{1}{1+\beta} F_{W} W_{x}=0,
$$

with $\beta$ a splitting parameter ( $\beta=\infty$ recovers the original conservative form). Here $\beta \neq-1$ and, for a perfect gas, $\beta>0$ or $\beta<\frac{\gamma}{1-\gamma}$. The theory only gives the range of $\beta$ and does not give any guidelines on how to choose $\beta$ for the particular flow. The vectors $F_{W}$ and $W$ can be cast as functions of the primitive variables $(\rho, u, p)$ and $\beta$. From the study of [83], $\beta$ is highly problem dependent. Multiplying the above equation by $W$ and integrating gives

$$
-(1+\beta)\left(W, U_{t}\right)=\beta\left(W, F_{x}\right)+\left(W, F_{W} W_{x}\right)=\beta\left(W, F_{x}\right)+\left(F_{W} W, W_{x}\right) .
$$

Integration-by-parts in space gives

$$
(1+\beta)\left(W, U_{t}\right)=-\left[W^{T} F_{W} W\right]_{a}^{b} .
$$

We thus obtain the estimate

$$
\begin{aligned}
\frac{d}{d t}\left(W, U_{W} W\right)= & \left(W_{t}, U_{W} W\right)+\left(W,\left(U_{W} W\right)_{t}\right)=\left(U_{t}, W\right)+\beta\left(W, U_{t}\right)= \\
& (1+\beta)\left(W, U_{t}\right)=-\left[W^{T} F_{W} W\right]_{a}^{b} .
\end{aligned}
$$


In order to have an energy estimate, the boundary term $\left[W^{T} F_{W} W\right]_{a}^{b}$ should be of the sign that makes the time derivative of the norm negative. For stability the entropy norm $\left(W, U_{W} W\right)$ should be bounded.

It is noted that the energy estimate can be shown to be identical with

$$
\frac{d}{d t} \int E(U)=\left[F^{E}\right]_{a}^{b}
$$

obtained by integrating the entropy equation (2.40) in space. It follows that, $W U_{W} W=(1+\beta) E(U)$ [73]. We can show that for the thermally perfect gas compressible Euler equations the mathematical entropy function can be a function of the physical entropy. Therefore, the resulting splitting was referred to as entropy splitting by Yee et al. [83].

\section{Discrete Analogue of the Continuum}

Standard stability guidelines for finite difference methods in solving nonlinear fluid flow equations are based on a linearized stability analysis. The linear stability criterion is applied to the frozen nonlinear problem at each time step and grid point. Most often the numerical BC (or boundary scheme), if needed, is not part of the stability analysis. The preceding section summarizes the historical perspective of entropy splitting of the fluid flow equation related to stable spatial finite discretization without paying attention to numerical BC. This section expands on stable finite difference methods that have a discrete analogue of the conditioned governing equations IBVPs. For ease of reference, "scheme" or more precisely "interior scheme" here generally refers to spatial difference schemes for the interior grid points of the computational domain, whereas "boundary scheme" is the numerical boundary difference operator for grid points near the boundaries. However, without loss of generality, we also adopt the conventional terminology of denoting "scheme" interchangeably as either the "combined interior and boundary scheme" or just the "interior scheme" within the context of the discussion.

The only tool needed to derive the norm estimates presented in the preceding section was integration-by-parts. One main point in this section is that the same norm estimates can be made for a semi-discrete difference approximations, if the differential operators are approximated by difference operators having the SBP property. Examples of this were shown in (2.16) and (2.21). Of course, the other estimates in the preceding section can be carried over to a semi-discrete approximation by use of SBP difference operators.

The discussion is divided into linearly stable and nonlinearly stable difference methods. It is important to point out that when solving the Navier-Stokes equations with complex viscous shock, shear-layer, and boundary layer and/or chemical reaction interactions, even after incorporating tools from recent de- 
velopments, the finite difference methods considered, although more rigorous than standard algorithms, are only linearly stable in a strict sense.

\subsection{Linearly Stable Difference Methods}

The most basic linear stability criterion is to investigate the behavior of the difference method when applied to a problem of constant coefficients and periodic boundaries. The Fourier symbol of the operator should be bounded. For higher than first-order methods, a complication is introduced by the numerical boundary treatment. Norm estimates, or normal mode analysis are normally employed (See Gustafsson et al. [24]). With these methods it is possible to prove stability for linear IBVPs. Difference operators having the SBP property with numerical BCs included have recently received some attention. See Strand, Olsson, and Nordström \& Carpenter [70, 53, 54, 55, 51]. As already discussed in Section 2, the idea with these operators is to have the property

$$
\left(D U_{j}, V_{j}\right)_{h}=-\left(U_{j}, D V_{j}\right)_{h}+V_{N} U_{N}-V_{1} U_{1},
$$

where $D$ is a difference operator approximating $d / d x$, including the accompanied boundary scheme. Typically $D$ is a standard centered operator in the interior of the computational domain, and has a special one-sided form near boundaries. The discrete scalar product is defined by (2.12), and is weighted by a positive definite matrix, $\sigma$. For the standard $L^{2}$-norm, $\sigma$ is the identity matrix. In [53,70], formulas for the norm and boundary modifications of $D$ are given which ensure the SBP property for operators up to order of accuracy eight. SBP satisfying numerical BCs are very different from the traditional numerical boundary treatment. For example, for a sixth-order central interior scheme, the SBP satisfying boundary schemes involved the modification of the central scheme at least 6 points from the boundary. The coefficients of these SBP boundary schemes are rational and irrational fractions. The coefficients of the boundary scheme are determined together with the weights, $\sigma$, in the scalar product, so that for each operator there is a particular scalar product in which the SBP property holds. With the SBP property, norm estimates of the difference approximation can be accomplished as the discrete analogue of the continuous energy estimate of the corresponding IBVP of the PDE.

\subsection{Nonlinearly Stable Difference Methods}

When using a linearly stable method on a nonlinear problem, nonlinear instabilities can appear. Instabilities can appear already for a linear problem with variable coefficients. For variable coefficient problems, it can be proved that numerical dissipation of not too high order will make the method stable. From a theorem by Strang it follows that a finite difference approximation of a nonlinear problem is stable, if the variable coefficient linearized approximation is 
stable, and the solution and the difference scheme are smooth functions. This is one reason for using numerical dissipation in practical flow simulation [24].

Before 1994, rigorous stability estimates for accurate and appropriate boundary schemes associated with fourth-order or higher spatial interior schemes were the major stumbling block in the theoretical development of combined interior and boundary schemes for nonlinear systems of conservation laws. Olsson [55] proved that an energy estimate can be established for second-order central schemes. To obtain a rigorous energy estimate for high order central schemes, one must apply the scheme to the split form of the inviscid governing equation. A discrete analogue of the continuum using a semi discrete approach can be written as

$$
\frac{d U_{j}(t)}{d t}=-\frac{\beta}{1+\beta} D F\left(U_{j}\right)-\frac{1}{1+\beta} F_{W}\left(U_{j}\right) D W_{j} .
$$

Here, $D$ is a difference operator, having the SBP property $[55,70]$. The estimate

$$
\frac{d}{d t}\left(W, U_{W} W\right)_{h}=-W_{N}^{T} F_{W}\left(W_{N}\right) W_{N}+W_{1}^{T} F_{W}\left(W_{1}\right) W_{1}
$$

in the discrete scalar product follows in the same way as for the PDE with indices 1 and $N$ the end points of the computational domain, and $h$ the grid spacing. Here the SBP satisfying difference operator, for example, consists of central difference interior operators of even order together with the corresponding numerical boundary operators that obey the discrete energy estimate. See Olsson and Strand for forms of the SBP boundary operators [55, 70]. As noted in Section 2, if odd order of the spatial discretizations are used, the difference operator $\mathrm{D}$ in (3.2) should be modified. In this case, $D$ should be employed on the non-conservative term, and $-D^{T}$, the negative adjoint of $D$ on the conservative term, i.e.,

$$
\frac{d U_{j}(t)}{d t}=-\frac{\beta}{1+\beta}\left(-D^{T}\right) F\left(U_{j}\right)-\frac{1}{1+\beta} F_{W}\left(U_{j}\right) D W_{j} .
$$

\subsection{SBP Difference Operators and Full Discretization}

There are two additional difficulties when applying the above semi-discrete SBP spatial discretization methods to realistic problems.

- How to impose given physical BCs without destroying the SBP property. For example, assume that we are given a boundary value $u\left(x_{1}\right)=g$ at the leftmost grid point of the domain at $j=1$. Applying the SBP operator at $j=2,3, \ldots, N$, and imposing $u_{1}=g$ would not lead to an estimate, since the one sided operator that should have been applied at 
$j=1$ is not applied. This in turn leads to additional non-zero terms in the scalar product (e.g., from the operator at $j=2$ ) which should have been canceled by terms from the operator at $j=1$.

- How to discretize in time. The semi-discrete estimates that show the norm decreases, will not necessarily lead to a decrease of the norm in a time discretized approximation. In many practical cases we do obtain a small increase in the norm for the time discrete problem. However, the stability is still greatly improved by use of entropy splitting and SBP operators, when compared with more standard schemes.

Several methods showing how to impose the physical $\mathrm{BC}$ have been proposed to overcome the first difficulty. Examples are the projection method [53] and the penalty method called "simultaneous approximation term" (SAT) $[10,51]$. For comparison of these methods, see $[69,48,29]$. The methods given in [10, 51] and [53] are based on linear properties and cannot be trivially generalized to the nonlinear Navier-Stokes equations, except for certain special cases. One such special case where the nonlinear case is covered by the theory involves imposing velocity zero on solid walls, where the simple approach of setting the velocity to zero after each time step coincides with the projection method in [53].

In addition, when time-dependent physical boundaries are involved, an additional complication arises, especially for multi-stage Runge-Kutta methods. If the time-dependent physical BC is not imposed correctly, the overall order of accuracy of the scheme cannot be maintained. Some systematic remedies are proposed but are rather complicated for variable coefficients and even more complicated for nonlinear problems. See [10, 8, 29].

For the full discretization of the problem, we should discretize in time in such a way that the discrete energy estimate also holds. The obvious solution would be to discretize in time in a skew-symmetric way, in a manner similar to the spatial discretization, e.g.,

$$
\begin{aligned}
\frac{\beta}{1+\beta} D_{t} U_{j}^{n} & +\frac{1}{1+\beta} U_{W}\left(W_{j}^{n}\right) D_{t} W_{j}^{n}= \\
& -\frac{\beta}{1+\beta} D F\left(U_{j}^{n}\right)-\frac{1}{1+\beta} F_{W}\left(W_{j}^{n}\right) D W_{j}^{n},
\end{aligned}
$$

where $D_{t}$ is a difference operator acting in the time direction. However, it turns out that the SBP property of the time difference quotient leads to a problem which is coupled implicitly in the time direction. To solve it we have to solve a nonlinear system of equations for all time levels in the same system, leading to an impractically large computational effort. Furthermore, numerical experiments shown in Sjögreen \& Yee [65] indicated that a bounded $L^{2}$ entropy norm $\left(W, U_{W} W\right)_{h}$ does not necessarily guarantee a well behaved numerical solution for long-time integrations. In other words, $L^{2}$ stability does not necessarily 
guarantee an accurate solution. In practical computations, the classical RungeKutta time discretizations using the method-of-lines approach (which we used for our numerical experiments) works well, but we have not been able to prove a time discrete entropy estimate for this method. In addition, numerical experiments shown in [65] indicate that the time discrete problem does not have a decreasing entropy norm for all values of $\beta$. Numerical experiments in Yee et al. $[83,61]$ also indicate the wide variations of the $\beta$ value for a full spectrum of flow problems. For example, if a constant $\beta$ is used for problems containing shock waves, a very large value of $\beta$ is needed. Otherwise divergent solutions or wrong shock location and/or shock strengths are obtained. In view of these findings, employing a constant $\beta$ (within the allowable range of $\beta$ ) throughout the entire computational domain appears not to be the best approach unless the flow problem is a simple smooth flow. Studies in $[83,63]$ indicate that the split form of the inviscid flux derivatives does help in minimizing the use of numerical dissipation. What is needed is adaptive control of the $\beta$ parameter from one flow region to another as well as from one physical problem to another. We caution that if the adaptation is not handled correctly, an abrupt switching of the $\beta$ can introduce spurious jumps in the numerical solutions. See [65] for the discussion.

In our computer code for the numerical experiment, we have implemented the sixth-order SBP operators by the projection and SAT methods given in [10, $51,53]$. They both perform satisfactorily, and no big difference in performance has been observed between them. See Section 6 for a 3-D compressible channel flow computation. We note that the majority of the physical boundaries of our viscous models are not time dependent, and the loss of spatial accuracy due to the multistage Runge-Kutta method is not a major concern.

\section{Adaptive Numerical Dissipation Control}

This section discusses the need for adaptive numerical dissipation controls in addition to conditioning the governing equations. An advanced numerical dissipation model for multiscale complex viscous flows is described.

The linear and nonlinear numerical dissipation (not filter) presently available is either built into the numerical scheme or added to the existing scheme. The built-in numerical dissipation schemes are, e.g., upwind, flux corrected transport (FCT), total variation diminishing (TVD), essentially non-oscillatory (ENO), weighted ENO (WENO), and hybrid schemes (e.g., those that switch between spectral and high-order shock-capturing schemes). The built-in nonlinear numerical dissipation in TVD, ENO and WENO schemes was designed to capture accurately discontinuities and high gradient flows while hoping to maintain the order of accuracy of the scheme away from discontinuities. These schemes have been shown to work well in a variety of rapidly developing shock-shock inter- 
actions that do not involve multiscale physics or long-time wave propagations. For multiscale physics that require low-dispersive errors, the amount of numerical dissipation built into these schemes is not optimal. In addition, analogous SBP theory for these schemes is not available. Moreover, they are more computationally expensive than standard high order centered schemes, and have severe limitations on the order of accuracy in the vicinity of the discontinuities and steep gradient regions. The inaccuracy of the numerical solutions can contaminate the entire flow field downstream [14]. Although, the amount of numerical dissipation is less than linear numerical dissipations, when applied to convection portions of viscous flows, it conflicts with the physical viscosity and can wash out the true physical steep gradient and/or turbulent structures. Aside from this fact, viscous reacting flows are even more difficult to simulate than non-reacting viscous flows. In the presence of numerical dissipations, even what is believed to be the optimal amount for non-reacting flows might have detrimental effects, e.g., wrong speeds of propagation and/or spurious traveling waves $[36,33,34]$.

There exist different specialized linear and nonlinear filters to post process the numerical solution after the completion of a full time step of the numerical integration. Since they are post processors, the physical viscosity, if it exists, is taken into consideration. The main design principle of linear filters is to improve nonlinear stability, to stabilize under-resolved grids [17] and to de-alias smooth flows, while the design principle of nonlinear filters is to improve nonlinear stability as well as accuracy near discontinuities. When discontinuities are present in the solution, linear filtering and/or entropy splitting might not be helpful or not applicable. The nonconservative terms of the entropy splitting might lead to inconsistent behavior at shocks/shears [83, 63, 65]. See, for example, $[22,17,18,76]$ for forms of linear filters, and see $[82,83,63]$ for forms of nonlinear filters. The use of the linear filter concept for smooth and/or turbulent flows has been employed for over two decades [76, 2, 37, 18]. For direct numerical simulation (DNS) and large eddy simulation (LES), there are additional variants of the linear filter approach. It was shown in Fischer \& Mullen [17] that adding an appropriate filter can stabilize the Galerkin-based spectral element method in convection-dominated problems. The Fischer \& Mullen numerical example illustrates the added benefit of the high-order linear filter. See Section 5.5 for a discussion.

For the last decade, CPU intensive high order schemes with built-in nonlinear dissipation have been gaining in popularity in DNS and LES for longtime integration of shock-turbulence interactions. Aside from the aforementioned short-coming of these built-in nonlinear dissipation high order schemes, their flow sensing mechanism is not sophisticated enough to clearly distinguish shocks/shears from turbulent fluctuations and/or spurious high-frequency oscillations. In $[82,83,63]$ it was shown that these built-in numerical dissipations 
are more dissipative and less accurate than the nonlinear filter approach of $[82,83,63]$ with a similar order of accuracy. It was also shown that these nonlinear filters can also suppress spurious high-frequency oscillations. However, a subsequent study of Sjögreen \& Yee [65] showed that the high order linear filter can sustain longer time integration more accurately than the nonlinear filter for low speed smooth flows. In other words, for the numerical examples that were examined in [65], the high order linear filter can remove spurious high-frequency oscillation producing nonlinear instability better than the second-order nonlinear filter. Higher than third-order nonlinear filters might be able to improve their performance or might outperform the high order linear filters in combating spurious high-frequency oscillations at the expense of more CPU time and added complexity near the computational boundaries. These findings prompted the design of switching on and off or blending of different filters to obtain the optimal accuracy of high order spatial difference operators as proposed in Yee et al. and Sjögreen \& Yee [83, 63]. The missing link of what was proposed in $[83,63]$ is an efficient, automated and reliable set of appropriate sensors that are capable of distinguishing key features of the flow for a full spectrum of flow speeds and Reynolds numbers.

We propose to enhance the conditioning of the equations with an advanced numerical dissipation model, which includes nonlinear sensors to detect shocks/shears and other small scale features, and spurious oscillation instability due to underresolved grids. Furthermore, we will use the detector to switch off the entropy splitting at shocks/shears and adjust the entropy splitting parameter with the aid of the local Mach number and Reynolds number in smooth regions as discussed earlier. The advanced numerical dissipation model can be used: (Option I) as part of the scheme, (Option II) as an adaptive filter control after the completion of a full time step of the numerical integration or (Option III) as a combination of Options I and II. For example, we can combine high order nonlinear dissipation (with sensor control) using Option I and nonlinear filter (with a different sensor control) using Option II.

The numerical experiments we have conducted so far concentrate on an adaptive procedure that can distinguish three major computed flow features to signal the correct type and amount of numerical dissipation needed in addition to controlling the entropy splitting parameter. The major flow features and numerical instability are (a) shocks/shears, (b) turbulent fluctuations, and (c) spurious high-frequency oscillations. It is important to not damp out the turbulent fluctuations. The procedure can be extended if additional refinement or classification of flow types and the required type of numerical dissipation is needed. There exist different detection mechanisms in the literature for the above three features. These detectors are not mutually exclusive and/or are too expensive for practical applications. We believe that the multiresolution wavelet approach proposed in Sjögreen \& Yee [63] is capable of detecting all 
of these flow features, resulting in three distinct sensors. If chosen properly, one multiresolution wavelet basis function might be able to detect all three features. For an optimum choice, one might have to use more than one type of wavelet basis functions but at the expense of an increase in CPU requirements. Some incremental studies into the use of entropy splitting and the application of these sensors were illustrated in $[83,63,64,66,65,50]$. The next section summarizes the development of adaptive filters for a special class of high order discretizations.

\section{High Order Filter Finite Difference Methods}

The adaptive numerical dissipation controls discussed in the preceding section are scheme independent. This section applies the adaptive ideas to high order central schemes. We first summarize the high order nonlinear filter schemes that were developed for shock/shear capturing. We then extend these filtering ideas to include more complex flow structures by blending more than one filter and sensing tool.

\subsection{ACM and Wavelet Filter Schemes for Discontinuity Capturing}

An alternative to linear filter and/or standard shock-capturing schemes for viscous multiscale and long-time wave propagation computations is the ACM (artificial compression method) and wavelet filter schemes described in [82, 63]. A high order centered base scheme together with the nonlinear dissipative portion of a shock-capturing scheme, activated by an ACM or wavelet sensor is used as the filter. Often an entropy split form of the inviscid flux derivatives is used. The idea of the ACM filter scheme is to have the spatially higher nondissipative scheme activated at all times and to add the full strength, efficient and accurate numerical dissipation only at the shock layers and steep gradients. Thus, it is necessary to have good detectors which flag the layers, and not the oscillatory turbulent parts of the flow field. While minimizing the use of numerical dissipation away from discontinuities and steep gradients, the ACM filter scheme consists of tuning parameters that are physical problem dependent. To minimize the tuning of parameters, new sensors with improved detection properties were proposed in Sjögreen \& Yee [63]. The new sensors are derived from utilizing appropriate non-orthogonal wavelet basis functions, and they can be used to completely switch off the extra numerical dissipation outside shock layers. The non-dissipative spatial base scheme of arbitrarily high order of accuracy can be maintained without compromising its stability at all parts of the domain where the solution is smooth. The corresponding scheme is referred to as the wavelet filter scheme. This nonlinear filter approach is particularly important for multiscale viscous flows. The procedure takes the 
physical viscosity and the reacting terms into consideration since only nondissipative high order schemes are used as the base scheme. In other words, numerical dissipations based on the convection terms are used to filter the numerical solution at the completion of the full step of the time integration at regions where the physical viscosity is inadequate to stabilize the high frequency oscillations due to the non-dissipative nature of the base scheme.

The method applied to the 2-D conservation law where $U$ is the conservative vector and $F$ and $G$ are the inviscid fluxes,

$$
U_{t}+F(U)_{x}+G(U)_{y}=0
$$

can be described as taking, e.g., one full time step by a Runge-Kutta method on the semi discrete system without or with entropy splitting, respectively, by

$$
\begin{gathered}
\frac{d U_{j, k}}{d t}=-D_{J} F\left(U_{j, k}\right)-D_{K} G\left(U_{j, k}\right), \\
\frac{d U_{j, k}}{d t}=-\frac{\beta}{1+\beta}\left[D_{J} F\left(U_{j, k}\right)+D_{K} G\left(U_{j, k}\right)\right] \\
-\quad \frac{1}{1+\beta}\left[F_{W}\left(U_{j, k}\right) D_{J} W_{j, k}+G_{W}\left(U_{j, k}\right) D_{K} W_{j, k}\right],
\end{gathered}
$$

where $D_{J}$ and $D_{K}$ are high order finite difference operators, acting in the $j$ - and $k$-direction respectively. They can be the SBP satisfying higher-order difference operators (e.g., sixth-order central scheme with SBP boundary schemes). We consider here a rectangular grid with grid spacing $\Delta x$ and $\Delta y$ and time step $\Delta t$. Denote a full Runge-Kutta step by

$$
\tilde{U}_{j, k}^{n+1}=R K\left(U_{j, k}^{n}\right)
$$

After the completion of a full Runge-Kutta step, a filter (post processing) step is applied leading to

$$
U_{j, k}^{n+1}=\tilde{U}_{j, k}^{n+1}-\lambda_{x}\left(\widetilde{F}_{j+1 / 2, k}-\widetilde{F}_{j-1 / 2, k}\right)-\lambda_{y}\left(\widetilde{G}_{j, k+1 / 2}-\widetilde{G}_{j, k-1 / 2}\right)
$$

with $\lambda_{x}=\Delta t / \Delta x$ and $\lambda_{y}=\Delta t / \Delta y$. The filter numerical fluxes $\tilde{F}_{j+1 / 2, k}$ and $\widetilde{G}_{j, k+1 / 2}$ act in the $j$ - and $k$-coordinate directions respectively, and are evaluated on the function $\widetilde{U}^{n+1}$. For example,

$$
\widetilde{F}_{j+1 / 2, k}=\frac{1}{2} R_{j+1 / 2} \Phi_{j+1 / 2}^{*}
$$

where $R_{j+1 / 2}$ is the right eigenvector matrix of the Jacobian of the inviscid flux $F$ evaluated at Roe's average state with the $k$ index suppressed. The $l$ th element of the filter flux $\Phi_{j+1 / 2}^{*}$ in the $x$-direction $\left(\phi_{j+1 / 2}^{l}\right)^{*}$ is a product of a sensor 
$\omega_{j+1 / 2}^{l}$ and a nonlinear dissipation $\phi_{j+1 / 2}^{l}, l=1,2,3,4$. With the omission of the $k$ index, it is of the form

$$
\left(\phi_{j+1 / 2}^{l}\right)^{*}=\omega_{j+1 / 2}^{l} \phi_{j+1 / 2}^{l} .
$$

For the ACM sensor, $\omega_{j+1 / 2}^{l}$ is a product of a physical dependent sensor coefficient and a gradient like detector. The nonlinear numerical dissipation $\phi_{j+1 / 2}^{l}$ can be obtained, from the dissipative portion of a TVD, ENO or WENO scheme. For example, the numerical flux $H_{j+1 / 2, k}$ of a second- or third-order TVD, ENO or WENO scheme can be written

$$
H_{j+1 / 2, k}=\frac{1}{2}\left(F_{j, k}+F_{j+1, k}\right)+\frac{1}{2} R_{j+1 / 2} \Phi_{j+1 / 2},
$$

with the first two terms corresponding to the flux average of a centered difference and $\Phi_{j+1 / 2}$ with elements $\phi_{j+1 / 2}^{l}$ being the numerical dissipation portion of the scheme.

For all the numerical experiments, the numerical dissipation portion of the Harten-Yee scheme is used. It has the form for the $j$-direction

$$
\phi_{j+1 / 2}^{l}=\frac{1}{2} Q\left(a_{j+1 / 2}^{l}\right)\left(g_{j+1}^{l}+g_{j}^{l}\right)-Q\left(a_{j+1 / 2}^{l}+\gamma_{j+1 / 2}^{l}\right) \widetilde{\alpha}_{j+1 / 2}^{l}
$$

with $Q(x)=\sqrt{x^{2}+\epsilon^{2}}$, the entropy satisfying remedy for the scheme with entropy correction parameter $\epsilon$ (not to be confused with the entropy splitting parameter). $a_{j+1 / 2}^{l}$ is the $l$ th characteristic speed evaluated at the Roe's average state in the $j$-direction. $\gamma_{j+1 / 2}^{l}$ is the modified characteristic speed and $g_{j}^{l}$ is a slope limiter which is a function of $\tilde{\alpha}_{j \pm 1 / 2}^{l}$, the jump in the characteristic variable in the $x$-direction.

A form of the ACM sensor $\omega_{j+1 / 2}^{l}$ proposed in [82] is

$$
\omega_{j+1 / 2}^{l}=\kappa \max \left(\theta_{j}^{l}, \theta_{j+1}^{l}\right)
$$

where

$$
\theta_{j}=\left|\frac{\left|\widetilde{\alpha}_{j+1 / 2}^{l}\right|-\left|\widetilde{\alpha}_{j-1 / 2}^{l}\right|}{\left|\widetilde{\alpha}_{j+1 / 2}^{l}\right|+\left|\widetilde{\alpha}_{j-1 / 2}^{l}\right|}\right| .
$$

See $[82,83]$ for details. It was shown in [63] that the method can be improved by letting the sensor $\omega_{j+1 / 2}^{l}$ be based instead on a regularity estimate obtained from the wavelet coefficients of the solution. The wavelet analysis gives an estimate of the so called local Lipschitz exponent $\alpha$. The dissipation is switched on for low $\alpha$ values, and switched off when $\alpha$ becomes large [63]. The wavelet analysis is more general and can be used to detect other features besides shocks/shears. The following gives a more detailed explanation. 


\subsection{Wavelet Sensor for Multiscale Flow Physics}

Wavelets were originally developed for feature extraction in image processing and for data compression. It is well known that the regularity of a function can be determined from its wavelet coefficients $[13,46,41]$ far better than from its Fourier coefficients. By computing wavelet coefficients (with a suitable set of wavelet basis functions), we obtain very precise information about the regularity of the function in question. This information is obtained just by analyzing a given grid function. No information about the particular problem which is solved is used. Thus, wavelet detectors are general, problem independent, and rest on a solid mathematical foundation.

As of the 1990's, wavelets have been a new class of basis functions that are finding use in analyzing and interpreting turbulence data from experiments. They also are used for analyzing the structure of turbulence from numerical data obtained from DNS or LES. See Farge [15] and Perrier et al. [57]. There are several ways to introduce wavelets. One standard way is through the continuous wavelet transform and another is through multiresolution analysis, hereafter, referred to as wavelet based multiresolution analysis. Mallet and collaborators $[41,42,43,44,45,46]$ established important wavelet theory through multiresolution analysis. See references $[72,71]$ for an introduction to the concept of multiresolution analysis. Recently, wavelet based multiresolution analysis has been used for grid adaptation (Gerritsen \& Olsson [20]), and to replace existing basis functions in constructing more accurate finite element methods. Here we utilize wavelet based multiresolution analysis to adaptively control the amount of numerical dissipation.

The wavelet sensor estimates the Lipschitz exponent of a grid function $f_{j}$ (e.g., the density and pressure). The Lipschitz exponent at a point $x$ is defined as the largest $\alpha$ satisfying

$$
\sup _{h \neq 0} \frac{|f(x+h)-f(x)|}{h^{\alpha}} \leq C,
$$

and this gives information about the regularity of the function $f$, where small $\alpha$ means poor regularity. For a $C^{1}$ wavelet function $\psi$ with compact support, $\alpha$ can be estimated from the wavelet coefficients, defined as

$$
w_{m, j}=<f, \psi_{m, j}>=\int f(x) \psi_{m, j}(x) d x,
$$

where

$$
\psi_{m, j}=2^{m} \psi\left(\frac{x-j}{2^{m}}\right)
$$

is the wavelet function $\psi_{m, j}$ on scale $m$ located at the point $j$ in space. This definition gives a so called redundant wavelet, which gives (under a few technical assumptions on $\psi$ ) a non-orthogonal basis for $L^{2}$. Theorem 9.2.2 in [13] 
states that if $\psi$ is $C^{1}$ and has compact support, and if the wavelet coefficients $\max _{j}\left|w_{m, j}\right|$ in a neighborhood of $j_{0}$ decay as $2^{m \alpha}$ as the scale is refined, then the grid function $f_{j}$ has Lipschitz exponent $\alpha$ at $j_{0}$. In practical computations, we have a smallest scale determined by the grid size. We evaluate $w_{m, j}$ on this scale, $m_{0}$, and a few coarser scales, $m_{0}+1, m_{0}+2$, and estimate the Lipschitz exponent at the point $j_{0}$ by a least square fit to the line [63]

$$
\max _{j \text { near }} \log _{0}\left|w_{m, j}\right|=m \alpha+c .
$$

Proper selection of the wavelet $\psi$ is very important for an accurate detection of the flow features. The result in [46, 45], which is used in [20], gives the condition that $\psi(x)$ should be the $k$ th-derivative of a smooth function $\eta(x)$ with the property

$$
\eta(x)>0, \quad \int \eta(x) d x=1, \quad \lim _{x \rightarrow \pm \infty} \eta^{(k)}(x)=0 .
$$

Then the result is valid for $0<\alpha<k$. A continuous function $f(x)$ has a Lipschitz exponent $\alpha>0$. A bounded discontinuity (shock) has $\alpha=0$, and a Dirac function (local oscillation) has $\alpha=-1$. Large values of $k$ can be used in turbulent flow so that large vortices or vortex sheets can be detected. Although the theorem above does not hold for $\alpha$ negative, a useful upper bound on $\alpha$ can be obtained from the wavelet coefficient estimate.

For the numerical experiments, the wavelet coefficient $w_{m, j}$ is computed numerically by a recursive procedure, which is a second-order B-spline wavelet or a modification of Harten's multi-resolution scheme [63]. We can express the algorithm as follows. Introduce the grid operators

$$
\begin{aligned}
& A f_{j}=\sum_{k=-p}^{q} d_{k} f_{j+k} \\
& D f_{j}=\sum_{k=-p}^{q} c_{k} f_{j+k}
\end{aligned}
$$

and its $m$ th level expanded versions

$$
\begin{aligned}
& A_{m} f_{j}=\sum_{k=-p}^{q} d_{k} f_{j+2^{m} k} \\
& D_{m} f_{j}=\sum_{k=-p}^{q} c_{k} f_{j+2^{m} k},
\end{aligned}
$$

where the integers $p$ and $q$ and the coefficients $c_{k}$ and $d_{k}$ are related to the chosen $\psi(x)$ and $\phi(x)$, and can be determined from them. Here $\phi(x)$ is the so called scaling function of the multiresolution wavelets.

The $m$ th level of wavelet coefficients can be written as

$$
w_{m, j}=\left\langle f, \psi_{m, j}\right\rangle=D_{m-1} A_{m-2} A_{m-3} \ldots A_{0} f_{j}, \quad m=1,2, \ldots
$$


Once the coefficients $d_{k}$ and $c_{k}$ are determined, the computation is a very standard application of grid operators. In practice, we only use $m_{0}=3$ to 5 . To be able to compute up to the boundary, we use one sided versions of the given operators. This seems to work well in practice, although it is not covered by the wavelet framework described above.

Detectors from the B-Spline Wavelet Basis Function. Developing the best suited wavelets that can characterize all of the flow features might involve the switching or blending of more than one mother wavelet $\psi(x)$ and scaling function $\phi(x)$, especially if one needs to distinguish turbulent fluctuations from shock/shear and/or spurious high frequency oscillations. The mother wavelet function used in [20] and described in detail in [46] meets some of our requirements. It is obtained from second order B-splines.

$$
\psi(x)=\left\{\begin{array}{ll}
0 & x>1 \\
-2(x-1)^{2} & 1 / 2<x<1 \\
-4 x(1-x)+2 x^{2} & 0<x<1 / 2 \\
-4 x(1+x)-2 x^{2} & -1 / 2<x<0 \\
2(x+1)^{2} & -1<x<-1 / 2 \\
0 & x<-1
\end{array} .\right.
$$

For this wavelet (5.20), there exists a scaling function, given by

$$
\phi(x)=\left\{\begin{array}{ll}
0 & x>2 \\
\frac{1}{2}(x-2)^{2} & 1<x<2 \\
-(x-1 / 2)^{2}+3 / 4 & 0<x<1 \\
\frac{1}{2}(x+1)^{2} & -1<x<0 \\
0 & x<-1
\end{array} .\right.
$$

The normalization is such that the integral of the scaling function above is equal to one. The functions above are standard, and can be found in [13]. The scaling function differs by a shift from the scaling function used in [20], but the important relations

$$
\begin{aligned}
& \phi(x)=\frac{1}{4} \phi(2 x+1)+\frac{3}{4} \phi(2 x)+\frac{3}{4} \phi(2 x-1)+\frac{1}{4} \phi(2 x-2) \\
& \psi(x)=\phi(2 x+1)-\phi(2 x)
\end{aligned}
$$

hold, and give the grid operators

$$
\begin{aligned}
A f_{j} & =\left(f_{j-1}+3 f_{j}+3 f_{j+1}+f_{j+2}\right) / 8, \quad j=2, \ldots, N-2 \\
D f_{j} & =\left(f_{j-1}-f_{j}\right) / 2 \quad j=2, \ldots, N .
\end{aligned}
$$

Note that this wavelet stencil is not symmetric. In general, the wavelet coefficients involve points from $p 2^{m_{0}-1}$ to $-q 2^{m_{0}-1}$, giving a stencil of totally $(p+q) 2^{m_{0}-1}+1$ points. 
Detectors from the Redundant Form of Harten Multiresolution Wavelet.

For the redundant form of Harten multiresolution wavelet there is more than one choice for the interpolation function. See Sjögreen [62] for a discussion. The exact form of the method for the computations in this article is

$$
\begin{aligned}
A f_{j} & =\left(f_{j-1}+f_{j+1}\right) / 2 \quad j=2, \ldots, N-1 \\
D f_{j} & =f_{j}-A f_{j} \quad j=2, \ldots, N-1 .
\end{aligned}
$$

The above choice was made in order to have a simple and efficient method. The stencil is narrower than for the B-spline formulas that were given previously. With the formula above we also get a symmetric stencil, which is more natural if the other parts of the computation, such as difference approximations of PDEs are done by symmetric formulas. Furthermore, symmetry makes periodic BCs somewhat easier to implement. Note that the absence of symmetry for either the scaling function or the wavelet can lead to phase distortion. This can be shown to be important in signal processing applications.

Multi-Dimensional Wavelets. The computation of multi-dimensional wavelets is quite expensive, especially in 3-D. A simple minded efficient way is to evaluate the wavelet coefficients dimension-by-dimension. This means that we get two set of wavelet coefficients $w_{m, j}^{x}(y)$ and $w_{m, k}^{y}(x)$, where now $(j, k)$ is the position and $m$ is the scale. The precise definition is

$$
\begin{aligned}
& w_{m, j}^{x}(y)=\int f(x, y) \psi_{m, j}(x) d x \\
& w_{m, k}^{y}(x)=\int f(x, y) \psi_{m, k}(y) d y
\end{aligned}
$$

Thus, the dimension-by-dimension approach involved only terms evaluated as finite differences in the $x$-direction and terms which are evaluated in the $y$ direction. We then use the $w_{m, j}^{x}(y)$ coefficients for the $x$-direction computation, and the $y$-coefficients for the $y$-direction computation.

Shock/Shear Wavelet Sensor. For the numerical experiments presented in the next section the wavelet sensor is obtained by computing a vector of the approximated Lipschitz exponent of a chosen vector function to be sensed with a suitable multiresolution non-orthogonal wavelet basis function. Here, "vectors or variables to be sensed" means the represented vectors or variables that are suitable for the extraction of the desired flow physics. The variables to be sensed can be the density, the combination of density and pressure, the characteristic variables, the jumps in the characteristic variables $\widetilde{\alpha}_{j+1 / 2}^{l}$, or the entropy variable vector $W([20,83])$. 
For example, if the characteristic variables are the chosen vector to be sensed by the wavelet approach, the sensor $\mathcal{S}_{j+1 / 2}^{l}$ can be defined as

$$
\mathcal{S}_{j+1 / 2}^{l}=\tau\left(\alpha_{j+1 / 2}^{l}\right),
$$

where $\alpha_{j+1 / 2}^{l}$ is the estimated Lipschitz exponent of the $l$ th characteristic component with $l=1,2,3,4$, the four characteristic waves. $\tau(\alpha)$ is a sensing function which decreases from $\tau(0)=1$ to $\tau(1)=0$ (for the aforementioned type of wavelets). Note that the $l$ th component of the estimated Lipschitz exponent $\alpha_{j+1 / 2}^{l}$ is not to be confused with the jump in the $l$ th characteristic variables $\widetilde{\alpha}_{j+1 / 2}^{l}$ in Section 5.1. We use $\mathcal{S}_{j+1 / 2}^{l}$ as the sensor to distinguish it from the ACM sensor $\omega_{j+1 / 2}^{l}$ in Section 5.1. Noted that the $k$ index is omitted (for the 2-D case) for simplicity.

If we instead base the exponent estimate on point centered quantities, we will use the sensor function

$$
\mathcal{S}_{j+1 / 2}^{l}=\max \left(\tau\left(\alpha_{j}^{l}\right), \tau\left(\alpha_{j+1}^{l}\right)\right) .
$$

If the exponent estimate is based on other quantities than the characteristic variables, (e.g., density and pressure), we use the switch

$$
\mathcal{S}_{j+1 / 2}=\max _{l} \mathcal{S}_{j+1 / 2}^{l},
$$

where the maximum is taken over all components of the waves used in the estimate. In this case, the switch is the same for all characteristic fields.

The function $\tau(\alpha)$ should be such that $\tau(0)=1$, and $\tau(1)=0$. Three options considered are

$$
\begin{aligned}
\tau(\alpha) & = \begin{cases}1 & \alpha<\alpha_{0} \\
0 & \alpha \geq \alpha_{0}\end{cases} \\
\tau(\alpha) & =\frac{1}{2}+\frac{1}{\pi} \arctan K\left(\alpha_{0}-\alpha\right) \\
\tau(\alpha) & =\max \left\{0, \min \left[1,(\alpha-1) /\left(\alpha_{0}-1\right)\right]\right\} .
\end{aligned}
$$

Here, $\alpha_{0}$ is a cut off exponent to be chosen. For the arctan function the values 0 and 1 are not attained, but we take the constant $K$ large enough so that the function is close to zero for $\alpha>1$, and close to one for $\alpha<0$. We have tried values for $K$ in the interval $[200,500]$. Alternatively, one can integrate the actual $\alpha$ value into the sensor function instead of using the same amount of numerical dissipation at the cut off exponent.

After some experimentation we have found that switching on the dissipation at the grid points where $\alpha<0.5$ works well, i.e.,

$$
\tau(\alpha)=\left\{\begin{array}{ll}
1 & \alpha<0.5 \\
0 & \alpha \geq 0.5
\end{array} .\right.
$$


In fact the method does not seem to be very sensitive to the exact value of cut off $\alpha_{0}$, (for $0.4 \leq \alpha_{0} \leq 0.6$ ) for all the test cases considered. Furthermore, the same cut off value, 0.5, works well for all problems we have tried in Section 6 (except for the vortex convection case, where $\alpha_{0}=0.0$ is used in conjunction with entropy splitting [83]). Experiments with smoothed step functions do not give very different results.

\subsection{Test Example of the Shock/Shear Wavelet Detectors}

This section shows the performance of the wavelet sensor using the dimensionby-dimension approach for a 2-D complex flow structure. It is important to note that the illustration involves only the feature extraction capability of the wavelet sensor on a given grid function. No dynamic behavior was involved (i.e., the numerical scheme is not part of the analysis). Figure 5.1 shows the computed density and pressure contours from a precomputed numerical simulation at $t=120$ with $\Delta t=0.12$ to be used as the two-dimensional discrete functions to be analyzed by the wavelet algorithm. The discrete functions represent a numerical data of a shock from the upper left corner, impinging on a horizontal shear layer in the middle of the domain. The shock is reflected from the lower wall boundary. For more details about the problem, see Yee et al. [82, 83].

Density

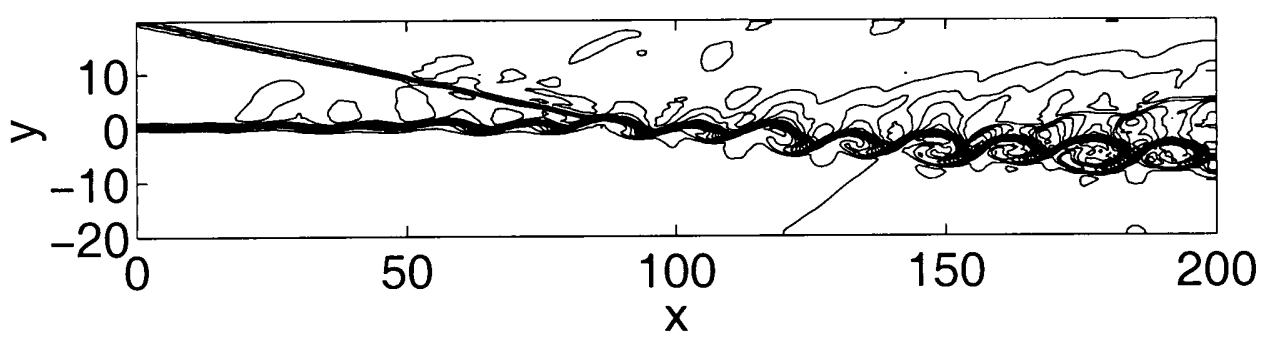

Pressure

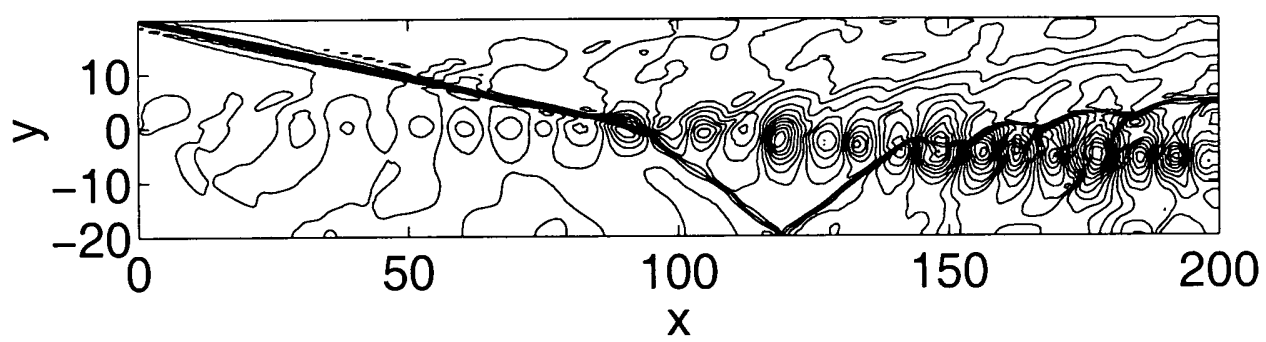

Fig. 5.1. 2-D Testing discrete function, (density and pressure contours at $t=120)$. 
Figure 5.2 shows contours of the estimated Lipschitz exponent $\alpha$ for the function in Fig. 5.1. The value $\alpha$ was computed here from three levels $\left(m_{0}=3\right)$ of the wavelet algorithm, using the wavelet coefficient

$$
w_{m, j, k}=\sqrt{\left(w_{m, j, k}^{x}\right)^{2}+\left(w_{m, j, k}^{y}\right)^{2}}
$$

where the one dimensional coefficients were computed by the multiresolution operators (5.24) in each coordinate direction. The coefficients were computed for the pressure. The top figure in Fig. 5.2 shows $\alpha$ contours on levels from 0.5 to 0.9 . The lower figure shows the corresponding sensor, a function which is one for $\alpha<0.5$ and zero otherwise. The wavelet sensor clearly captures the shock and the shear layer. The low $\alpha$ at the upper boundary to the right is probably due to mildly unstable BCs at the upper boundary.

\section{Alpha contours [0.5 0.9]}

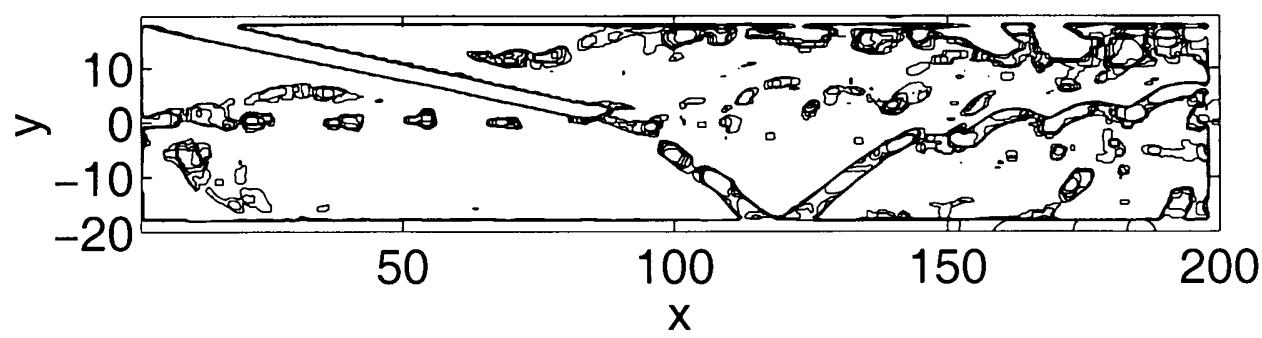

Sensor, contour at 0.5

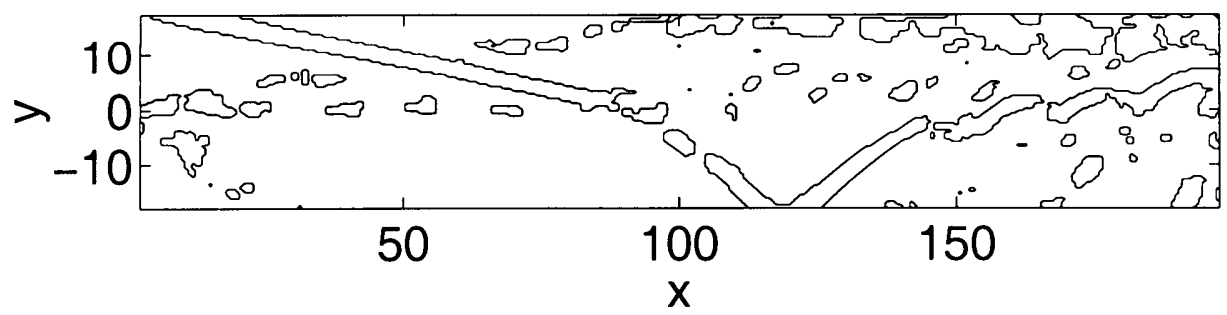

Fig. 5.2. Top: $\alpha$ contours $0.5 \leq \alpha \leq 0.9$; Bottom: sensor contour at $\alpha=0.5$. by the RH-wavelet.

\subsection{Blending of Different Filters}

The nonlinear filters for the ACM or wavelet shock/shear capturing nonlinear filter might not be sufficient for (a) time-marching to steady state and (b) spurious high frequency oscillations due to insufficient grid resolution and nonlinear 
instability away from discontinuities, especially for turbulent and large-eddy simulations. This Section discusses the blending of other filters with these shock/shear filters.

In classical CFD codes, a second order accurate base method is used together with two constant strength linear numerical dissipation terms. One linear fourthorder dissipation is used everywhere except near shocks/shears/steep-gradients to remove nonlinear instabilities. It does not affect the second order accuracy of the base scheme. The second dissipation term is a second-order linear dissipation, which affects the order of accuracy, but is only switched on near discontinuities, and/or steep unresolved gradients using a gradient sensor. The sensor used cannot distinguish the different flow features distinctly and is not accurate enough for turbulent statistics and long-time acoustic computations, unless extreme grid refinement is employed.

In analogy with the aforementioned classical methods, a more advanced numerical dissipation model with improved flow feature extraction sensors for high order central schemes is proposed. Here, we consider a dissipation model with two parts. One part is a nonlinear filter ([82]) and the second part is a high order linear numerical dissipation term modified at boundaries to become a semi-bounded operator, see $[67,65]$. The wavelet dissipation control sensor developed in [63] is used as the flow feature detector.

Time-Marching to Steady State. For time-marching to steady state one usually needs to add fourth-order linear dissipation to a second-order spatial differencing scheme (Beam and Warming (1976)). For the present schemes using characteristic filters, in addition to the nonlinear shock/shear filter, one might need to add a sixth-order linear dissipation to a fourth-order spatial base scheme and an eighth-order linear dissipation to a sixth-order spatial base scheme in regions away from shocks for stability and convergence. Let $L_{d}$ be such an additional filter operator. The two ways of incorporating the $L_{d}$ operator are options I and II discussed in Section 4.

To minimize the amount of dissipation due to $L_{d}$ in the vicinity of shock waves, there should be a switching mechanism $\kappa_{d}$ to turn off the $L_{d}$ operator in the vicinity of shock waves. The $L_{d}$ operator can be applied to the conservative, primitive or characteristic variables. The simplest form is to apply $L_{d}$ to the conservative variables. Alternatively, since all of the work in computing the average states and the characteristic variables is done for the shock-capturing filter operator, one can apply the $L_{d}$ operator to the characteristic variables. In this case, the switching mechanism $k_{d}$ can be a vector so that it is more in tune with the nonlinear shock detector using the approximate Riemann solver. For example, one can set $\kappa=0$ for the linearly degenerate fields and blend a small amount of $\kappa_{d}$ to remove spurious noise generated by the lack of nonlinear 
filters. This blending of the nonlinear shock/shear filter with the $L_{d}$ operator can be applied to time-accurate computations as well.

Suppression of Spurious High Frequency Oscillations. The nonlinear shock/shear filters might not be able to remove spurious high frequency oscillations effectively unless sufficient fine grid points are used. For the suppression of unphysical high frequency oscillations due to insufficient grid resolution and nonlinear instability away from discontinuities, higher-order spectral-like filters (Vichnevetsky (1974), Lele (1994), Alpert (1981), Visbal and Gaitonde (1998), Gaitonde and Visbal (1999)) might be needed at the locations where the value of the shock/shear sensor is very small or zero. If spectral-like filters are needed, a proper blending of nonlinear shock/shear filters with spectral-like filters should be applied. In this case, we can use the same procedures as the time-marching to the steady state except the $L_{d}$ operator should be replaced with the spectral-like filters (for compact central schemes).

\section{An Adaptive Numerical Dissipation Model for Shock-Turbulence Inter-} actions. Assume a sufficient grid is used for the problem and scheme in question, and that the scale of turbulent fluctuations is larger than the spurious high-frequency oscillations. Below we present a filter model under these assumptions. If the scale of the turbulent fluctuation is in similar scale as the high-frequency oscillations, a different wavelet with a turbulent fluctuation sensor should be added. For example, for a sixth-order central spatial base scheme, we define the 1-D filter numerical flux of the numerical dissipation operator as $H_{j-1 / 2}^{d}$.

$$
H_{j-1 / 2}^{d}=S_{j-1 / 2} F_{j-1 / 2}^{*}+d_{j}\left[1-S_{j-1 / 2}\right]\left(h^{6} D_{-}\left(D_{+} D_{-}\right)^{3} U_{j},\right.
$$

here $S_{j-1 / 2}$ is a switch computed as described in Section 5.2.4, and $F_{j-1 / 2}^{*}$ is the flux function corresponding to the dissipative portion of a shock-capturing scheme (e.g., second order accurate TVD scheme) [82]. The first part of the filter stabilizes the scheme at shock/shear locations. The second part is an eighthorder linear filter which improves nonlinear stability away from shock/shear locations. Analogous eighth-order filters can be used if a sixth-order compact spatial base scheme is used $[18,76]$. We switch on the high order part of the filter when we switch off the nonlinear filter. The physical quantity (e.g., local Mach number) can be used to determine the $d_{j}$ parameter of this high order dissipation term.

To further increase stability properties, it is possible to use the sensor to switch on and off the entropy splitting and adjust the value of the entropy splitting parameter according to flow type and region. For the 1-D shock/turbulence interactions to be presented in the next section, however, we believe a constant 
$\beta=1$ away from the shock waves is sufficient. After the completion of a full time step computation using the sixth-order base scheme (denoting the solution by $\widetilde{U}_{j}$ ), we filter this solution by

$$
U_{j}^{n+1}=\widetilde{U}_{j}+\frac{\Delta t}{h}\left[H_{j+1 / 2}^{d}-H_{j-1 / 2}^{d}\right]
$$

Here the filter numerical fluxes $H_{j \pm 1 / 2}^{d}$ are evaluated at $\widetilde{U}$.

\subsection{Spurious Numerical Solutions and Instability Due to Under-Resolved Grids}

There has been much discussion on verification and validation processes for establishing the credibility of CFD simulations $[68,7,75,21,52]$. Since the early 1990s, many of the aeronautical and mechanical engineering related reference journals mandated that any accepted articles in numerical simulations (without known solutions to compare with) need to perform a minimum of one level of grid refinement and time step reduction. On the other hand, it has become common to regard high order schemes as more accurate, reliable and requiring less grid points. The danger comes when one tries to perform computations with the coarsest grid possible while still hoping to maintain numerical results sufficiently accurate for complex flows and, especially, data-limited problems. On one hand, high order methods when applied to highly coupled multidimensional complex nonlinear problems might have different stability, convergence and reliability behavior than their well studied low order counterparts, especially for nonlinear schemes such as TVD, MUSCL with limiters, ENO, WENO, and spectral elements and discrete Galerkin. See for example references $[23,74,6,49,78,81,80,79]$. On the other hand, high order methods involve higher operation counts per grid and systematic grid convergence studies can be time consuming and prohibitively expensive. At the same time it is difficult to fully understand or categorize the different nonlinear behavior of finite discretizations, especially at the limits of under-resolution when different types of numerical (spurious) bifurcation phenomena might occur, depending on the combination of grid spacings, time steps, initial conditions (ICs) and numerical treatments of BCs as well as the nonlinear stability of the scheme in question. 
$(256,16) \alpha=0.0, t=1.0$

(a)

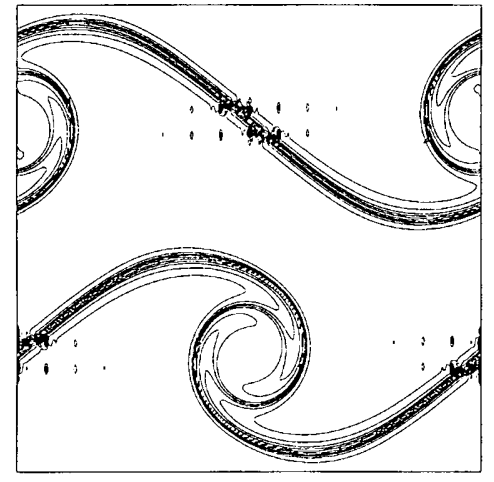

$(256,8) \alpha=1.0, t=1.5$

(c)

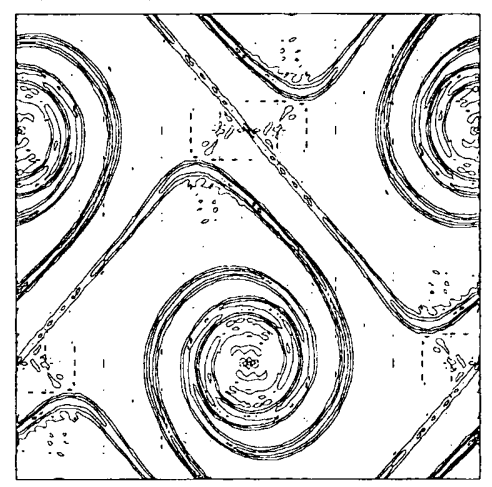

$(1024,8) \alpha=0.05, t=1.2$

(e)

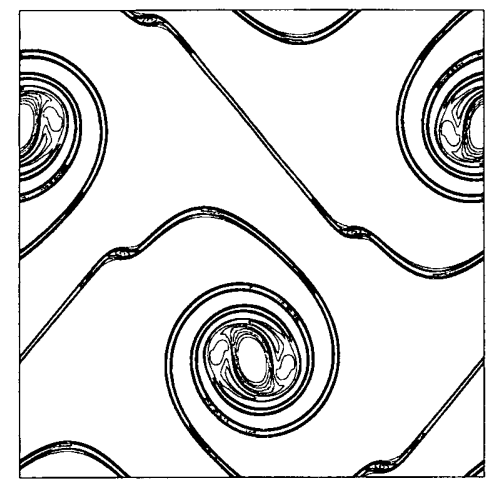

$(256,16) \alpha=0.3, t=1.5$

(b)

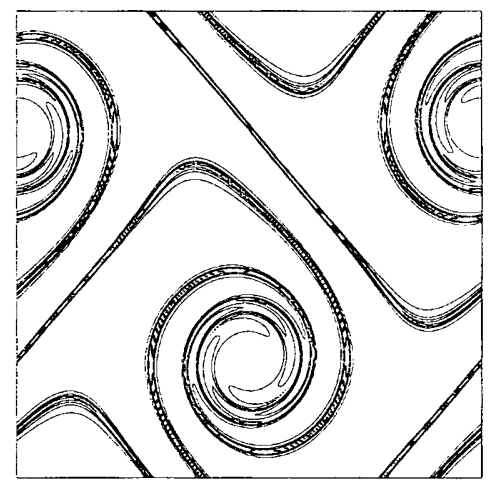

$(256,8) \alpha=0.3, t=1.5$

(d)

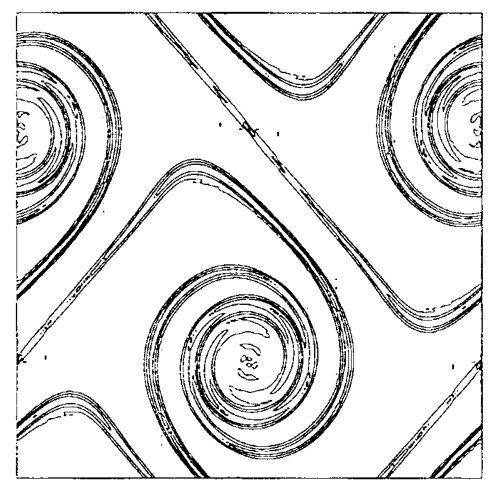

$(256,16) \alpha=0.05, t=1.2$

(f)

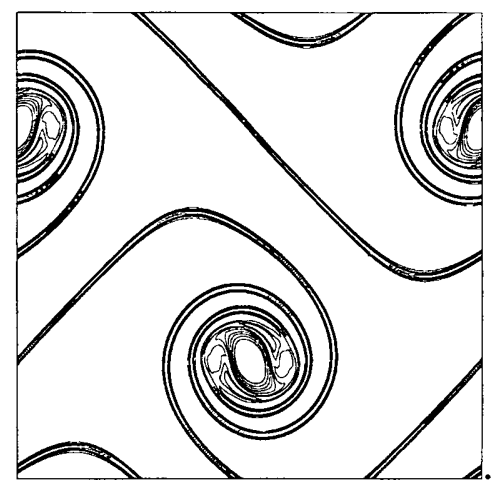

Fig. 5.3. Spectral element solutions of doubly periodic shear layer roll-up problem with different $(E, N)$ pairings and filter strengths $\alpha$ : (a-d) thick shear layer case $(\rho=30)$, (e-f) thin shear layer case $(\rho=100)$. 
Due to the difficulty in analysis, the effect of under-resolved grids and the nonlinear behavior of available spatial discretizations are scarcely discussed in the literature. Here, an under-resolved numerical simulation, according to Brown \& Minion, is one where the grid spacing being used is too coarse to resolve the smallest physically relevant scales of the chosen continuum governing equations that are of interest to the numerical modeler. Before the nineties, it was common in DNS to avoid the use of numerical dissipations. It was standard practice to refine the grid not just to resolve the multiscale physics but also to overcome nonlinear instability instead of employing numerical dissipation or filters. In certain cases, the grid is finer than what is needed to resolve the smallest scale. This section illustrates the situation where the necessity of finer grid can be overcome by the use of an appropriate filter and still be able to obtain an accurate and stable solution with a much coarser grid.

Brown and Minion [6, 49] studied the effects of under-resolved grids by considering the shear layer roll-up problem that arises when the Navier-Stokes equations are solved in the unit square with doubly-periodic BCs with ICs given by

$$
\begin{gathered}
u=\left\{\begin{array}{ll}
\tanh (\rho(y-0.25)) & \text { for } \mathrm{y} \leq 0.5 \\
\tanh (\rho(0.75-y)) & \text { for } \mathrm{y}>0.5
\end{array},\right. \\
v=0.05 \sin (2 \pi x) .
\end{gathered}
$$

In $[6,49]$, the behavior of several difference methods was considered. These difference methods include a Godunov projection method, a primitive variable ENO method, an upwind vorticity stream-function method, centered difference methods of both a pressure-Poisson and vorticity stream-function formulation, and a pseudospectral method. They demonstrated that all these methods produce spurious, non-physical vortices. While these extra vortices might appear to be physically reasonable, they disappear when the mesh is refined.

Figure 5.3 shows filter-based spectral element results for the problem (5.34) as computed by Fischer and Mullen [17]. The spectral element method is characterized by the discretization pair $(E, N)$, where $E$ is the number of quadrilateral elements and $N$ is the order of the tensor-product polynomial expansion within each element. This filter presented in [17] is designed to stabilize the $P N 2$ spectral element method at high Reynolds numbers. The $P N 2$ method, introduced by Maday and Patera [40], is a consistent approximation to the Stokes problem which employs continuous velocity expansions of order $N$ and discontinuous pressure expansions of order $N-2$. The discretizations in Fig. 5.3a 5.3e consist of a 16 array of elements, while Fig. $5.3 \mathrm{f}$ consists of a $32 \times 32$ array. Here, $\alpha$ denotes the spectral filter coefficient (not to confused with the Lipschitz exponent or the jump in the characteristics in Sections 5.1 and 5.2), with $\alpha=0$ corresponding to no filtering. The time step size is $\Delta t=0.002$ in all cases, corresponding to CFL numbers in the range of 1 to 5 . Without filtering, Fischer 
and Mullen were not able to simulate this problem at any reasonable resolution. Figure 5.3a illustrates the result prior to blow up for the unfiltered case with $(E, N)=\left(16^{2}, 16\right)$, which has a resolution corresponding to an $n \times n$ grid with $n=256$. Unfiltered results for $N=8(n=128)$ and $N=32(n=512)$ are similar. Filtering with $\alpha=0.3$ yields dramatic improvement for $n=256$ (Fig. 5.3b) and $n=128$ (Fig. 5.3d). Though the so-called full projection with filter strength $\alpha=1$ is stable, the partial filtering of $(\alpha<1)$ gives smoother results and is preferable. The cases in 5.3e and 5.3f correspond to the difficult "thin" shear layer case of [6] and show the benefits of high-order discretizations. Both cases correspond to a resolution of $n^{2}=256^{2}$. In Fig. 5.3e, this is attained with $(E, N)=\left(16^{2}, 16\right)$, while in Fig 5.3f, $(E, N)=\left(32^{2}, 8\right)$. Although both results are stable (due to the filter), Fig 5.3f reveals the presence of spurious vortices that are absent in the higher-order case.

\section{Numerical Examples}

This section illustrates the power of entropy splitting, the difference in performance of linear and nonlinear (with sensor controls) filters and the combination of both types of filters with adaptive sensor controls. We use the same notation as in [82, 83, 64]. The artificial compression method (ACM) and wavelet filter schemes using a second-order nonlinear filter with sixth-order spatial central interior scheme for both the inviscid and viscous flux derivatives are denoted by ACM66 and WAV66. See [82, 83, 64] for the forms of these filter schemes. The same scheme without filters is denoted by CEN66. The scheme using the fifth-order WENO for the inviscid flux derivatives and sixthorder central for viscous flux derivatives is denoted by WENO5. Computations using the standard fourth-order Runge-Kutta temporal discretization are indicated by appending the letters "RK4" as in CEN66-RK4. ACM66 and WAV66 use the Roe's average state and the van Leer limiter for the nonlinear numerical dissipation portion of the filter. The wavelet decomposition is applied in density and pressure, and the maximum wavelet coefficient of the two components is used. The nonlinear numerical dissipation is switched on wherever the wavelet analysis gives a Lipschitz exponent [63] less than 0.5. Increasing this number will reduce oscillations, at the price of reduced accuracy (see [63] for other possibilities). Computations using entropy splitting are indicated by appending the letters "ENT" as in WAV66-RK4-ENT. Computations using an eighth-order linear dissipation filter are indicated by appending the letters "D8" as in WAV66-RK4-D8. In order not to introduce additional notation, inviscid flow simulations are designated by the same notation, with the viscous terms not activated. 


\section{Isentropic Vortex Evolution \\ (Horizontally Convecting Vortex, vortex strength $\hat{\beta}=5$ )}

Freestream:

$$
\left(u_{\infty}, v_{\infty}\right)=(1,0) ; p_{\infty}=\rho_{\infty}=1
$$

IC: Perturbations are added to the freestream (not in entropy)

$$
\begin{aligned}
(\delta u, \delta v) & =\frac{\hat{\beta}}{2 \pi} e^{\frac{1-\gamma^{2}}{2}}\left(-\left(y-y_{0}\right),\left(x-x_{0}\right)\right) \\
\delta T & =-\frac{(\gamma-1) \hat{\beta}^{2}}{8 \gamma \pi^{2}} e^{1-r^{2}} \\
r^{2} & =\left(x-x_{0}\right)^{2}+\left(y-y_{0}\right)^{2}
\end{aligned}
$$

Computational Domain \& Grid Size:

$$
\begin{aligned}
& 0 \leq x \leq 10 \&-5 \leq y \leq 5 \\
& 80 \times 79 \text { Uniform grid } \\
& \text { Periodic BC in } x \& y
\end{aligned}
$$

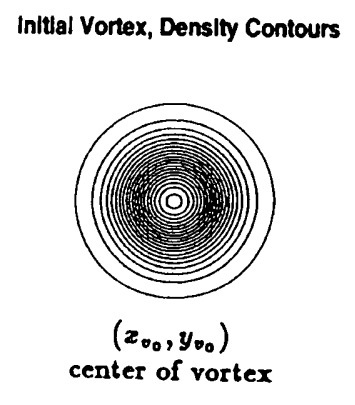

Figure 6.1. Vortex convection problem description.

\subsection{A 2-D Vortex Convection Model [82, 83, 63, 65]}

The onset of nonlinear instability of long-time numerical integration, the benefit of the entropy splitting and the difference in performance of linear and nonlinear numerical dissipations in improving nonlinear stability for a horizontally convecting vortex (see Fig. 6.1) can be found in in $[82,83,63,65]$. We summarize the results here.

To show the onset of nonlinear instability, the 2-D perfect gas compressible Euler equations are approximated by CEN66-RK4 with periodic BCs imposed using a $80 \times 79$ grid with the time step $\Delta t=0.01$. Since this is a pure convection problem, the vortex should convect without any distortion if the numerical scheme is highly accurate and non-dissipative. Although CEN66RK4 is linearly stable, the test problem is nonlinear and instability eventually sets in. Almost perfect vortex preservation is observed for up to 5 periods of integrations (5 times after the vortex has convected back to the same position time $=50$ ). Beyond 5 periods the solution becomes oscillatory, and blows up before the completion of 6 periods. The blow up is associated with an increase in entropy [65]. If we instead use the entropy-split form of the approximation (CEN66-RK4-ENT) with a split parameter $\beta=1$, almost perfect vortex preservation for up to 40 periods can be obtained. The computation remains stable for up to 67 periods before it breaks down. The time history of the $L^{2}$ entropy norm 


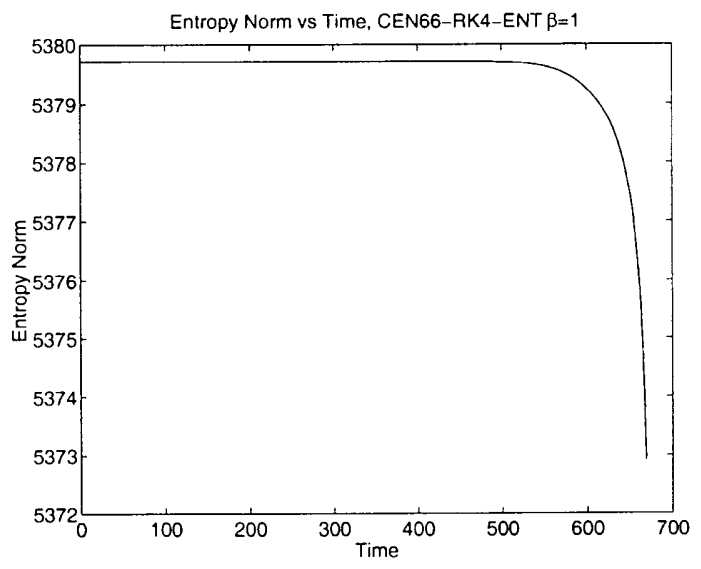

Figure 6.2. Entropy norm history of CEN66-ENT: entropy split parameter $\beta=1$ and $80 \times 79$ grid.

and density contours of the IC and the computed solution after 5, 10, 30, 50 and 67 periods using CEN66-RK4-ENT is shown in Figs. 6.2 and 6.3. The norm is decreasing, although the instabilities break down the solution after 67 periods. Using the second-order nonlinear filter without splitting (ACM66-RK4 or WAV66-RK4), the solution remains stable beyond 67 periods. However, the numerical solution gradually starts to diffuse after 20 periods. If we use the nonlinear filter in conjunction with entropy splitting (ACM66-RK4-ENT or WAV66-RK4-ENT), almost perfect vortex preservation can be obtained for up to 120 periods using a split parameter $\beta=1$ [83].

The density contours of the solution after $5,10,200$ and 300 periods for the un-split $(\beta=\infty)$ computation using the eighth-order linear dissipation (CEN66-RK4-D8) are shown in Fig. 6.4. The linear dissipation $\left(-d h^{7}\left(D_{+} D_{-}\right)^{4} U_{j}\right)$ with grid spacing $h$ was added to the sixth-order base scheme to discretize the convection terms. The parameter $d$ is a given constant $(d=0.002)$ and is scaled with the spectral radius of the Jacobian of the flux function, and $D_{+}$ and $D_{-}$are the forward and backward difference operators, respectively. This numerical dissipation is applied as part of the scheme and not as a post processing filter. The computation can be run for 300 periods without breakdown. However, serious degradation of accuracy occurs after 250 periods. For this particular problem, the CEN66-RK4-D8 out performed the ACM66-RK4-ENT and WAV66-RK4-ENT using $\beta=1$. Perhaps using a higher than third-order nonlinear filter might improve the performance of the ACM66-RK4-ENT and WAV66-RK4-ENT at the expense of an increase in CPU. 

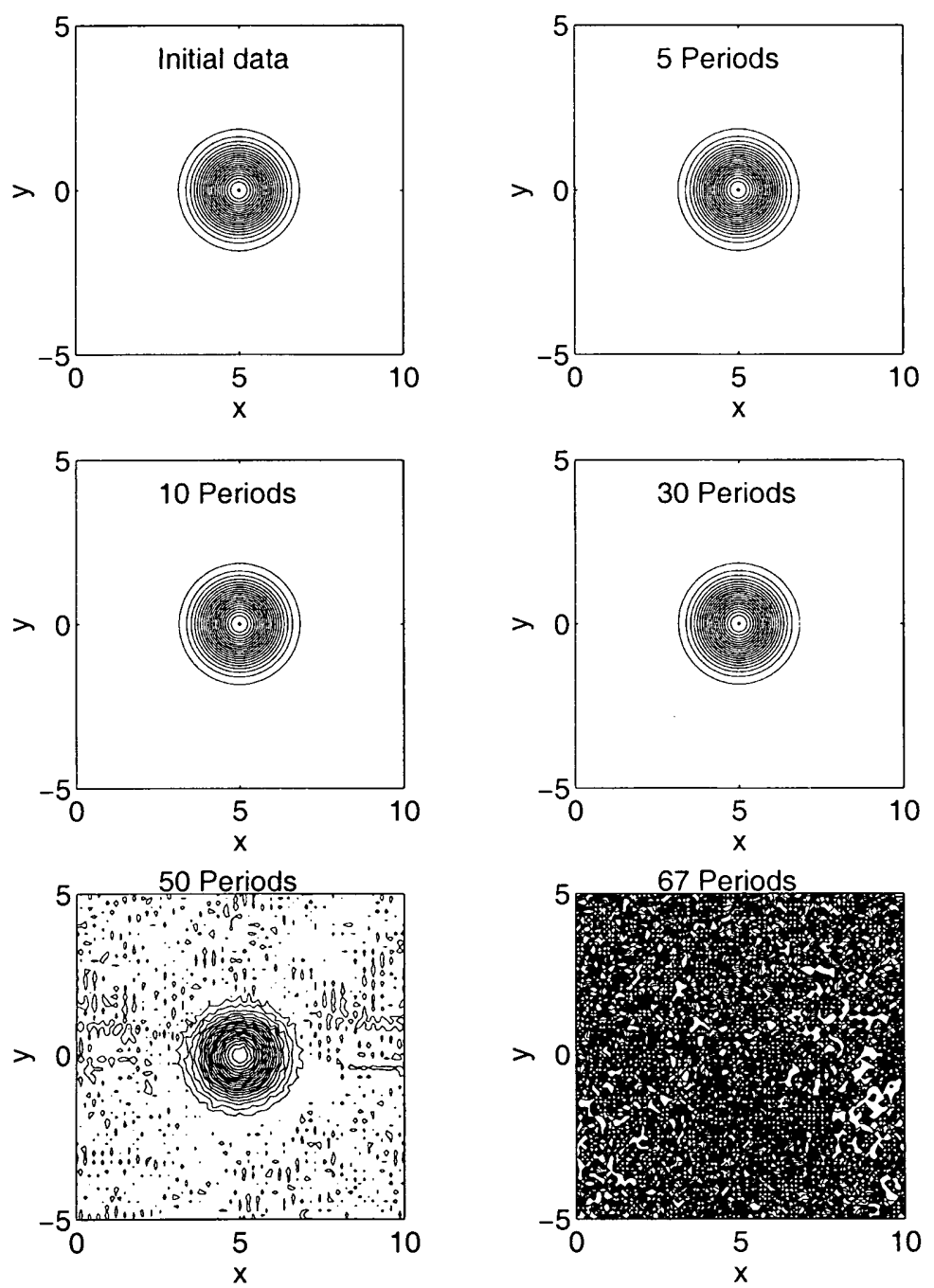

Figure 6.3. Density Contours of CEN66-ENT: entropy split parameter $\beta=1$ and $80 \times 79$ grid. 

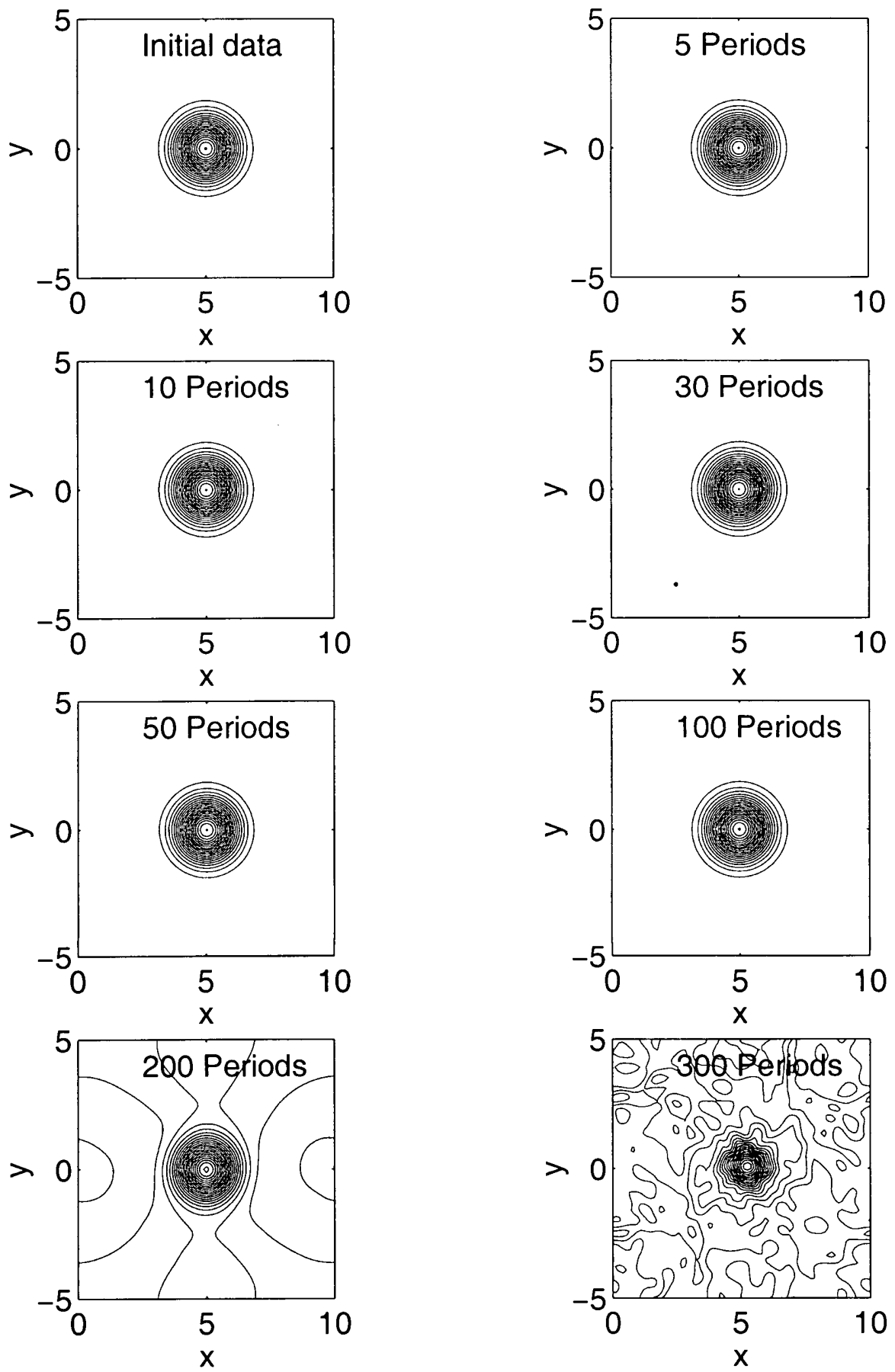

Figure 6.4. Density contours of CEN66-RK4-D8 using $80 \times 79$ grid. 


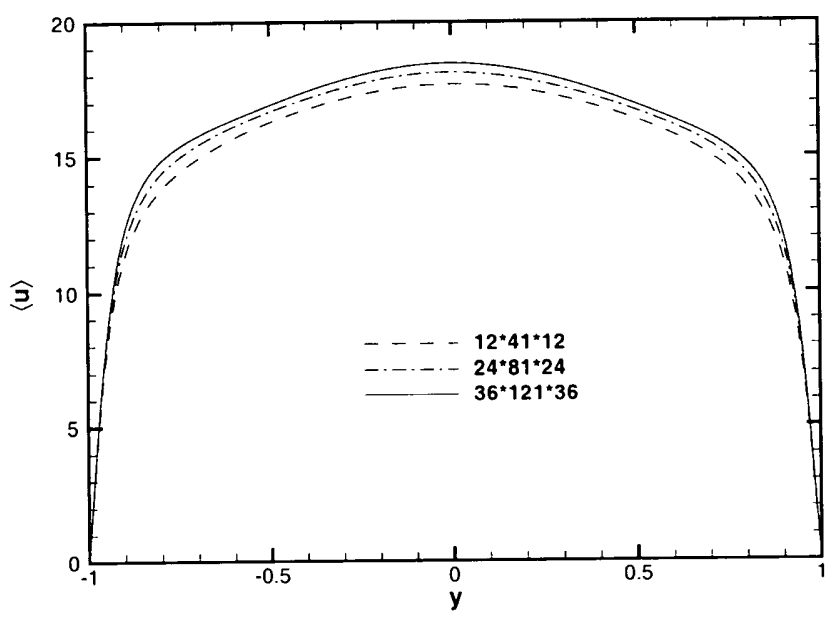

Figure 6.5. Mean velocity profiles

\subsection{DNS of 3-D Compressible Turbulent Channel Flow} [61]

This numerical example illustrates the power of entropy splitting for DNS computations. To obtain accurate turbulent statistics, very long-time integration and highly accurate methods are required for this DNS computation. The computation employed the SBP-satisfying boundary difference operator [9] with the fourth-order central interior scheme applied to the split form of the inviscid flux derivatives CEN44-RK4-ENT with $\beta=4$, and a Laplacian viscous formulation. The fluid mechanics of this 3-D wall bounded isothermal compressible turbulent channel flow has been studied in some detail by Coleman et al. [11]. They showed that the only compressibility effect at moderate Mach numbers comes from the variation of fluid properties with temperature. They used a uniform body force term to drive the flow, but recommended the constant pressure gradient approach which was adapted by Sandham et al. [61].

Grid refinement study. A simplified case is taken in which the fluid properties (viscosity and thermal conductivity) are held constant and the computational box size is kept small. The latter is justified as a method of reducing cost as the gross turbulence statistics are relatively insensitive to the computation box size, so long as the domains are still significantly larger than the minimal domains on which turbulence can be sustained. A Mach number of 0.1 is chosen, based on friction velocity and sound speed corresponding to the fixed wall temperature. 


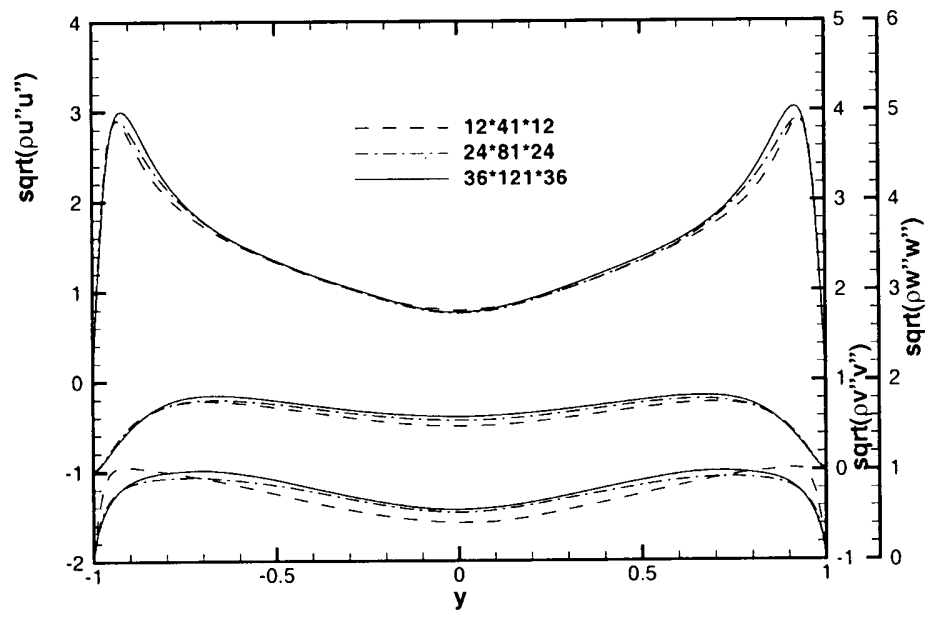

Figure 6.6. Effect of grid refinement on normal stresses. The top curves relate to the left scale, the middle to the right scale and the lowest to the furthest right scale.

Channel half width $h$, friction velocity $u_{\tau}$, wall temperature and bulk (integrated) density are the normalizing quantities for non-dimensionalization with a Reynolds number of 180 . Together with the constant property assumption, this choice of Mach number means that results can reasonably be compared to results from previous incompressible flow calculations. The computations were carried out at a fixed CFL=2.0. They were started with artificial ICs and first run to time $t=50$, by which time dependence on the ICs is lost. Statistics were accumulated over the time interval $t=200$ to $t=300$.

Three grids $12 \times 41 \times 12,24 \times 81 \times 24$ and $36 \times 121 \times 36$ were considered. The largest number in each case corresponds to the direction normal to the wall (y). The computational box has non-dimensional length 3 in the (streamwise) $x$ direction, 1.5 in the (spanwise) $z$-direction and 2 in the $y$-direction. The $x$ - and $z$-directions have periodic BCs with uniform grid spacing. In the $y$-direction, the grid is stretched according to

$$
\frac{y}{h}=\frac{\tanh \left(c_{\eta} \eta\right)}{\tanh c_{\eta}}
$$

with $\eta$ uniformly distributed on $[-1,1], c_{\eta}=1.7$. The ratio of grid points in each direction was chosen so that all directions have roughly the same degree of resolution of the relevant turbulence microscales in each direction. Figure 6.5 shows the mean flow velocity, Fig. 6.6 the root mean normal stresses and Fig. 6.7 the stress profiles across the channel. Angle brackets \langle\rangle denote averages 


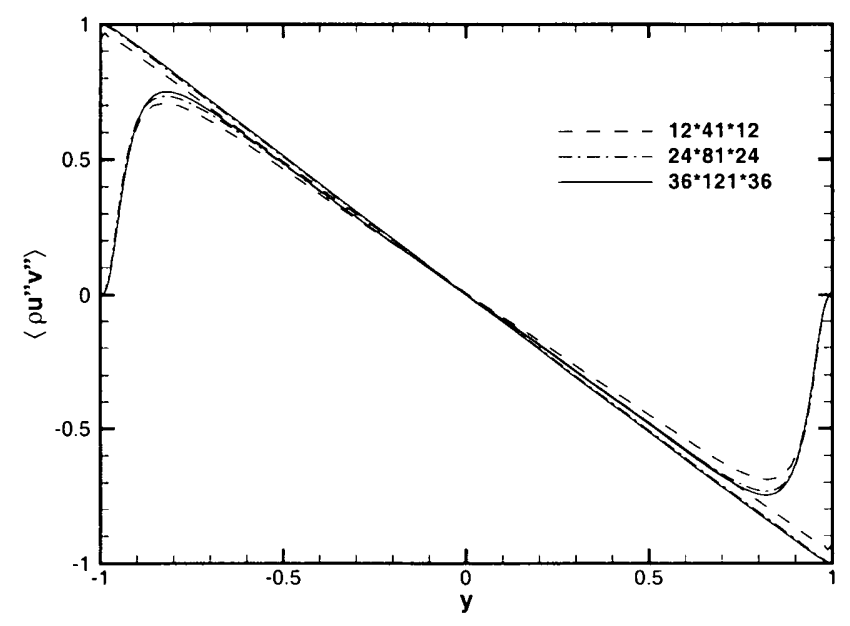

Figure 6.7. Effect of grid refinement on turbulent shear stress (curve falling to zero at the walls). The total stress (straight lines, non-zero at walls) is also shown.

over the homogeneous spatial directions and time while in the usual notation double primes denote deviations from mass-weighted (Favre) averages. The convergence is not uniform across the channel but the change from medium to fine grid is smaller than the change from coarse to medium grid. A comparison of the rms quantities with an incompressible flow simulation on the same size computational box (Z. Hu, private communication) is shown in Fig. 6.8. Here we compare the $36 \times 121 \times 36$ fourth-order compressible simulation with a $32 \times 81 \times 32$ fully spectral incompressible simulation using the method described in [59]. As expected, good agreement is found, as expected for this Mach number ( 0.1 based on friction velocity or 1.8 based on centerline velocity).

The convergence of various global measures can be found in Table 1 of [61] for the three grids. For the pressure gradient and Reynolds number specified, the velocity gradient at the wall should be 180 , the difference away from this being an error of the simulation. Here $R e_{\tau}$ is the Reynolds number based on $u_{\tau}$, the mean density at the wall $\left\langle\rho_{w}\right\rangle$ and the mean viscosity $\left\langle\mu_{w}\right\rangle$ at the wall. For the finest grid the resolution in wall units (a common check on resolution in DNS) is $\Delta_{x}^{+}=15$ and $\Delta_{z}^{+}=7.5$ and approximately 10 points are in the sublayer $y^{+}<10$. The simulations demonstrate a robustness down to very coarse resolutions, comparable with the best incompressible turbulent flow solvers incorporating de-aliasing and skew-symmetric formulation of the convective terms. Without the use of the entropy splitting of the inviscid flux derivatives and without the use of the Laplacian viscous formulation, the CEN44-RK4 


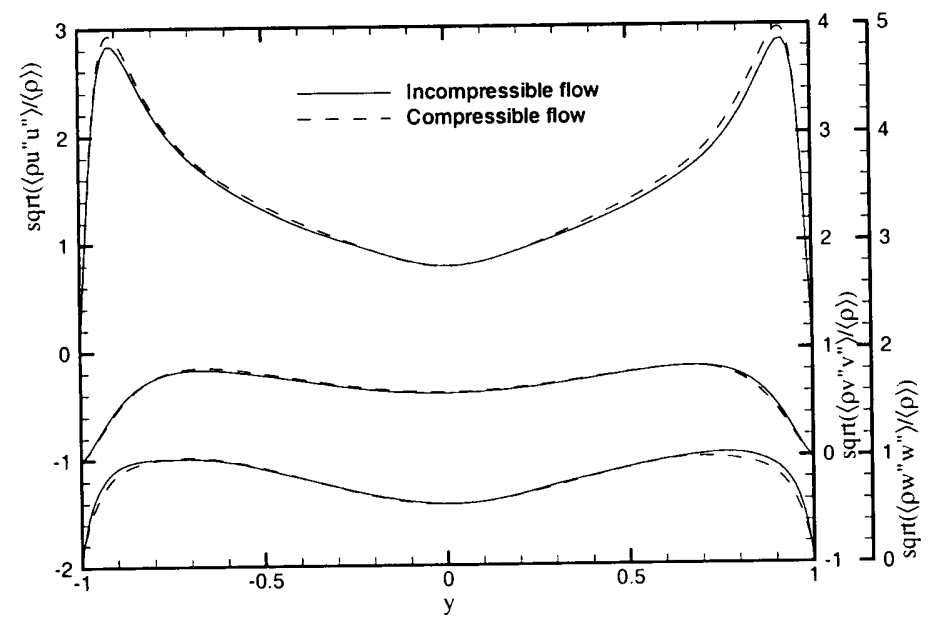

Figure 6.8. Comparison of weighted turbulence quantities on a $36 \times 121 \times 36$ grid with an incompressible flow simulation on a $32 \times 32 \times 81$ grid.

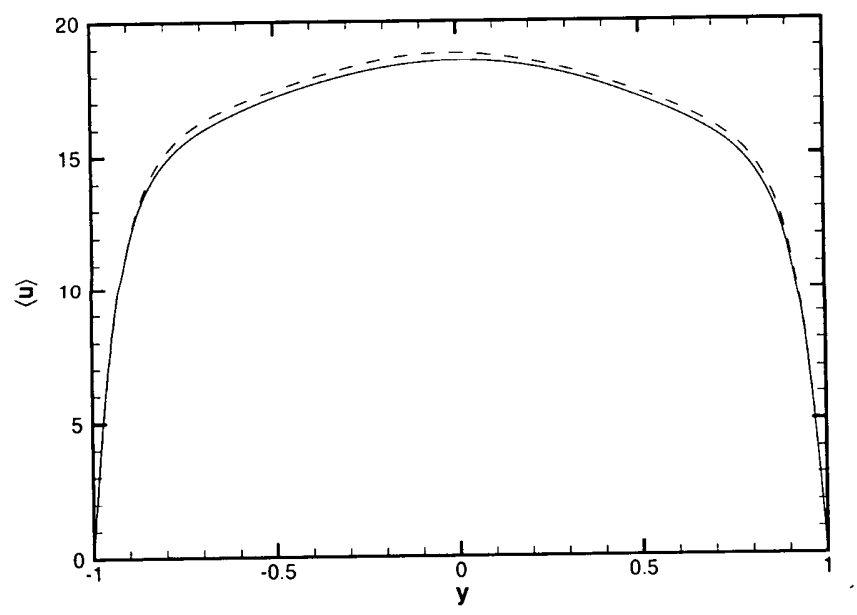

Figure 6.9. Mean velocity profile, comparing current simulation (dashed line) with Coleman et al. $[11]$ (solid line).

(un-split) solutions for the same CFL number, diverge for all three grids before meaningful turbulence statistics can be obtained. 


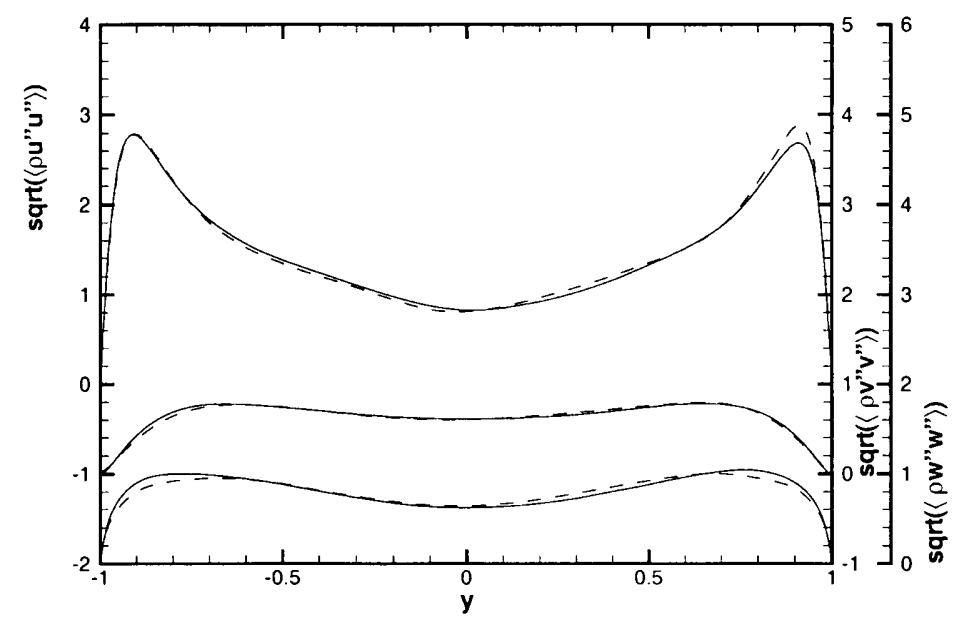

Figure 6.10. Root mean normal turbulent stresses, comparing current simulation (dashed line) with Coleman et al.[11](solid line). The top curves relate to the left scale, the middle to the right scale and the lowest to the furthest right scale.

Comparison with Coleman et al.. Coleman et al. [11] carried out comparable simulations in their study of the effects of Mach number on turbulence statistics. This section shows the simulation of their case $R e_{\tau}=190$ and $M_{\tau}=0.095$ with a uniform body force term together with variable fluid properties (power-law temperature dependence of the viscosity with exponent 0.7 and fixed Prandtl number $\mathrm{Pr}=0.7$ ). With the variable viscosity there is a need to use a larger computational box size than was used in the preceding section, since turbulence structures become larger as the viscosity is reduced (the wall is cold relative to the bulk flow). We chose to use a box of size $6 \times 2 \times 3$, i.e., twice as large in $x$ and $z$ as in the preceding section. This size is still somewhat lower than that of Coleman et al., who used a box of size $4 \pi \times 2 \times 4 \pi / 3$. A computational grid of $60 \times 141 \times 60$ was used, giving $\Delta x^{+}=19$ and $\Delta z^{+}=9.5$. These are comparable to those used by Coleman et al. (16.6 and 10.0 respectively). There were 12 points in the sublayer $\left(y^{+}<10\right)$.

For this simulation a parallel implementation was used, which illustrated the excellent parallel scaling of the method on a Cray T3E-1200E computer $(90 \%$ efficiency for a $240^{3}$ benchmark on 256 processors and continued good scaling up to 768 processors, as reported in Ashworth et al. [3]). The simulation presented here used 32 processing elements.

Table 1 shows a summary of the output from the simulation. Data from Coleman et al. have been re-normalized for comparison with the current simu- 


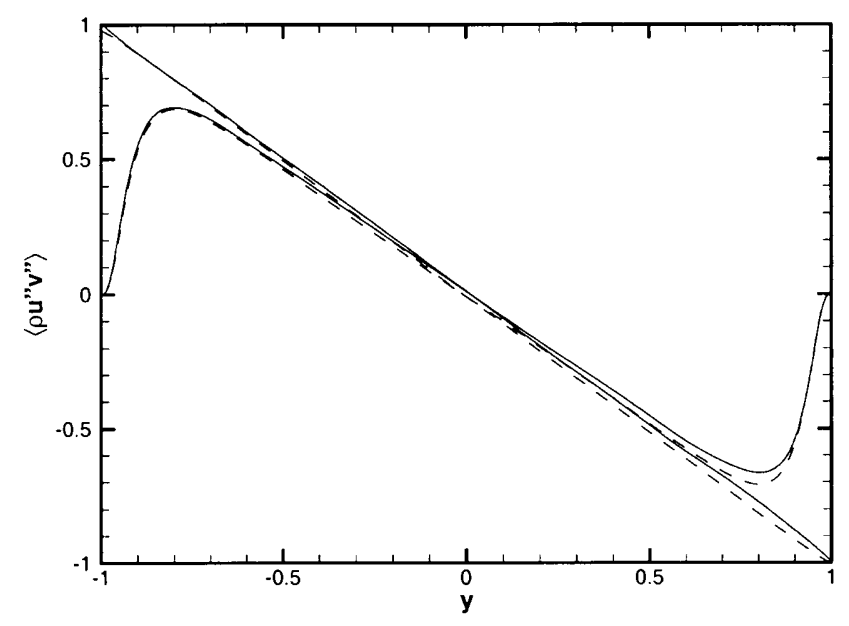

Figure 6.11. Turbulent and total shear stresses, comparing current simulation (dashed line) with Coleman et al.[11](solid line).

Table 1. Comparison of centerline velocity $U_{c}$, bulk velocity $U_{b}$, wall shear stress $\left\langle\mu \frac{d u}{d y}\right\rangle_{w}$ and shape factor $H$ with Coleman et al.[11]

\begin{tabular}{ccccc}
\hline Simulation & $U_{c}$ & $U_{b}$ & $\left\langle\mu \frac{d u}{d y}\right\rangle_{w}$ & $\mathrm{H}$ \\
\hline Current & 18.9 & 16.3 & 190.3 & 1.66 \\
\hline Coleman et al. & 18.5 & 15.9 & 189.5 & 1.65 \\
\hline
\end{tabular}

lation results. Figures 6.10 and 6.11 show the shear stress and rms turbulence fluctuations, while Fig. 6.9 shows the mean velocity profile. Overall a good agreement is obtained illustrating the good performance of the method for a resolution comparable to that of a spectral method. Good turbulence kinetic energy budgets have also been obtained [38].

We note that for this well-studied problem with accurate turbulent flow databases for comparison, we can safely conclude that entropy splitting in conjunction with the Laplacian formulation calculations was able to obtain stable and fairly accurate solutions using coarse to moderate grid sizes without added numerical dissipation or filters. Unlike the spectral method, this high order method can be efficiently extended to general geometries [77]. For the same 3-D problem, the finite difference formulation of the WENO5 is more than six 
times as expensive, yet more diffusive than the present scheme using the same temporal discretization.

The numerical methods are currently being applied to several practical problems. Alam \& Sandham [1] have studied shock-free transition to turbulence near the leading edge of an aerofoil, while Lawal \& Sandham [35] have used the above method in conjunction with the high order nonlinear filter scheme from Yee $e t$ al. [82] to study transitional shock boundary layer interaction in flow over a bump. These practical applications have been run without the need for changes to the numerical method and hence are leading to some confidence that the developments presented here are generally applicable for DNS of compressible turbulent flow .

For the performance of ACM66-RK4, WAV66-RK4 and WENO5-RK4 on a spatially or a time-developing mixing layer problem containing shock waves, see $[83,63]$.

\subsection{Computational Aeroacoustics Applications (CAA) [50]}

This numerical example illustrates the applicability of the entropy splitting to CAA for low Mach number flows. The numerical prediction of vortex sound has been an important goal in CAA since the noise in turbulent flow is generated by vortices. To verify our numerical approach for CAA, the Kirchhoff vortex is chosen for the numerical test. The Kirchhoff vortex is an elliptical patch of constant vorticity rotating with constant angular frequency in irrotational flow. The acoustic pressure generated by the Kirchhoff vortex is governed by the 2D Helmholtz equation, which can be solved analytically for almost circular Kirchhoff vortices using separation of variables. See [50] and references cited therein for details. The difficulty with this test case is the large gradient of the acoustic pressure adjacent to the Kirchhoff vortex.

The perturbation form of the entropy split 2D Euler equations in conjunction with a fourth-order linear filter operator CEN66-RK4-ENT-D4 was applied to Kirchhoff vortex sound at low Mach number using a high order metric evaluation of the coordinate transformation. SBP operators using the SAT method of implementing the time-dependent physical $\mathrm{BC}$ was employed. Due to the large disparity of acoustic and stagnation quantities in low Mach number aeroacoustics, the split Euler equations are formulated in perturbation form to minimize numerical cancellation errors.

A very accurate numerical solution with a relatively coarse grid was obtained using CEN66-RK4-ENT-D4 compared with the un-split (CEN66-RK4D4), and un-filter cases CEN66-RK4, CEN22-RK4 and CEN44-RK4. The extra CPU due to the use of the split form of the inviscid flux derivatives is more than compensated by the improved accuracy and stability of the numer- 
ical simulation, especially near regions of large gradients. For this weakly nonlinear test case that does not require long time integration, the amount of filter needed, although very small, is still important. Higher order filter operators and the formulation of the numerical BCs and filter operators in terms of the entropy variables to satisfy a discrete energy estimate in a nonlinear sense will be considered in the future.

\subsection{Multiscale Complex Unsteady Viscous Compressible Flows [64, 66]}

Extensive grid convergence studies using WAV66-RK4 and ACM66-RK4 for two complex highly unsteady viscous compressible flows are given in $[64,66]$. The first flow is a 2-D complex viscous shock/shear/boundary-layer interaction. This is the same problem and flow conditions studied in Daru \& Tenaud [12]. The second flow is a supersonic viscous reacting flow concerning fuel breakup. More accurate solutions were obtained with WAV66-RK4 and ACM66-RK4 than with WENO5-RK4, which is nearly three times as expensive. To illustrate the performance of these nonlinear filter schemes, the first model is considered. The ideal gas compressible full Navier-Stokes equations with no slip BCs at the adiabatic walls are used. The fluid is at rest in a $2-\mathrm{D}$ box $0 \leq x, y \leq 1$. A membrane with an initial shock Mach number of 2.37 located at $x=1 / 2$ separates two different states of the gas. The dimensionless initial states are

$$
\rho_{L}=120, \quad p_{L}=120 / \gamma ; \quad \rho_{R}=1.2, \quad p_{R}=1.2 / \gamma
$$

where $\rho_{L}, p_{L}$ are the density and pressure respectively, to the left of $x=1 / 2$, and $\rho_{R}, p_{R}$ are the same quantities to the right of $x=1 / 2 . \gamma=1.4$ and the Prandtl number is 0.73 . The viscosity is assumed to be constant and independent of temperature, so Sutherland's law is not used. The velocities and the normal derivative of the temperature at the boundaries are set equal to zero. This is done by leaving the value of the density obtained by the one sided difference scheme at the boundary unchanged, and updating the energy at the boundary to make the temperature derivative equal to zero.

At time zero the membrane is removed and wave interaction occurs. An expansion wave and a shock are formed initially. Then, a boundary layer is formed on the lower boundary behind the right going waves. After reflection, the left going shock wave interacts with the newly formed boundary layer, causing a number of vortices and lambda shocks near the boundary layer. Other kinds of layers remain after the shock reflection near the right wall. The complexity of this highly unsteady shock/shear/boundary-layer interactions increases as the Reynolds number increases.

As an illustration, we show here the difficult case of Reynolds number $R e=1000$. The computations stop at the dimensionless time 1 when the reflected shock wave has almost reached the middle of the domain, $x=1 / 2$. 
The numerical results discussed here are at time 1 with a uniform Cartesian grid spacing as described by Daru and Tenaud. Due to symmetry, only the lower half of the domain is used in the computations; symmetry BCs are enforced at the boundary $y=1 / 2$. Figure 6.12 shows the comparison of a secondorder MUSCL using a second-order Runge-Kutta method (MUSCL-RK2) with WAV66-RK4, ACM66-RK4 and WENO5-RK4 using a $1000 \times 500$ grid. Comparing with the converged solution of WAV66-RK4 and ACM66-RK4 using $3000 \times 1500$ (see bottom of figure) and $4000 \times 2000$ grids (see [64]), one can conclude that WAV66-RK4 exhibits the most accurate result among the $1000 \times 500$ grid computations. We note that, for this Reynolds number, the unsteady problem is extremely stiff, requiring very small time steps and very long-time integrations before reaching the dimensionless time of 1 .

\subsection{1-D Shock-Turbulence Interactions Using the Adaptive Numerical Dissipation Model}

The dissipative model (5.32) is used to solve a simple, yet difficult, 1-D compressible inviscid shock-turbulence interaction problem with initial data consisting of a shock propagating into an oscillatory density. The initial data are given by

$$
\left(\rho_{L}, u_{L}, p_{L}\right)=(3.857143,2.629369,10.33333)
$$

to the left of a shock located at $x=-4$, and

$$
\left(\rho_{R}, u_{R}, p_{R}\right)=(1+0.2 \sin (5 x), 0,1)
$$

to the right of the shock where $u$ is the velocity. Fig. 6.13 show the comparison between a second-order MUSCL-RK2 with a sixth-order central scheme and the aforementioned numerical dissipation model using RK4 as the time discretization (WAV66-RK4-D8). The parameter $d=0.002$ is scaled with the spectral radius of the Jacobian of the flux function. Note that the eighth-order dissipation is a filter, and is different from the CEN66-D8 used in Section 6.3 where the dissipation is part of the scheme. The solution using a second-order uniformly non-oscillatory (UNO) scheme on a 4000 uniform grid is used as the reference solution (solid lines on the first three sub-figures). The bottom of the right figures show the density and Lipschitz exponent distribution for the WAV66-RK4-D8 using 400 grid points. Comparing our result with the most accurate computation found in the literature for this problem, the current approach is highly efficient and accurate using only 800 grid points without grid adaptation or a very high order shock-capturing scheme. For the present computation, the WAV66-RK4-D8 consumed only slightly more CPU than the second-order scheme MUSCL-RK2. With the eighth-order dissipation filter 


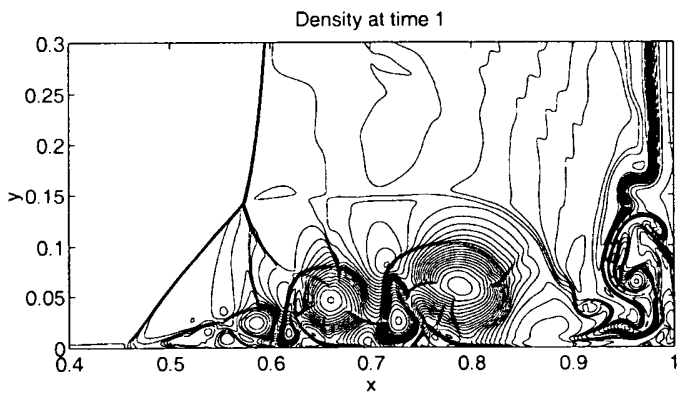

(a) MUSCL-RK2, $1000 \times 500$ grid

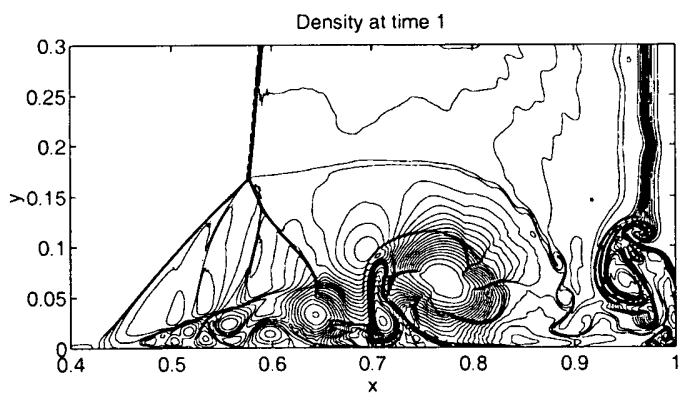

(c) WAV66-RK4, $1000 \times 500$ grid

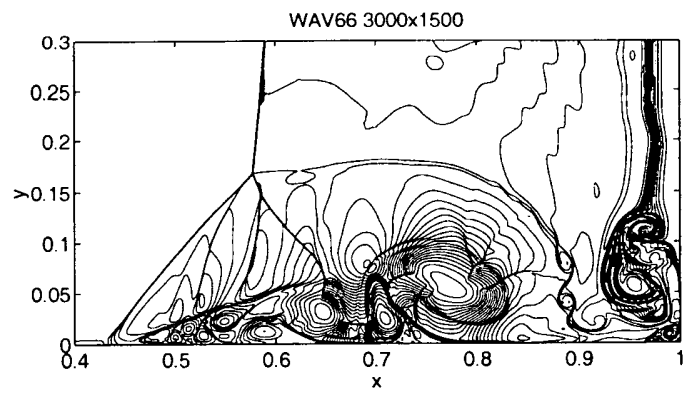

(e) WAV66-RK4, $3000 \times 1500$ grid

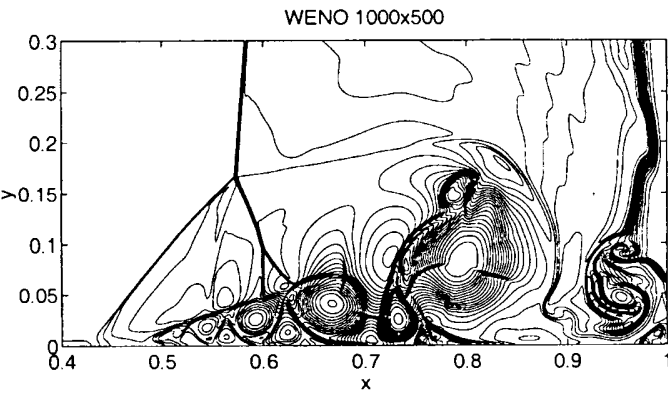

(b) WENO5-RK4, $1000 \times 500$ grid

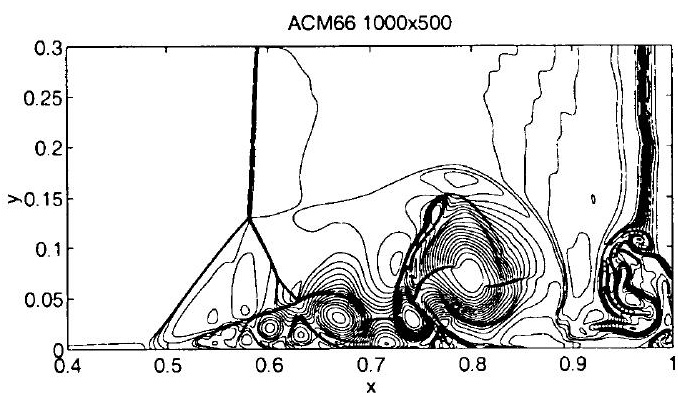

(d) ACM66-RK4, $1000 \times 500$ grid

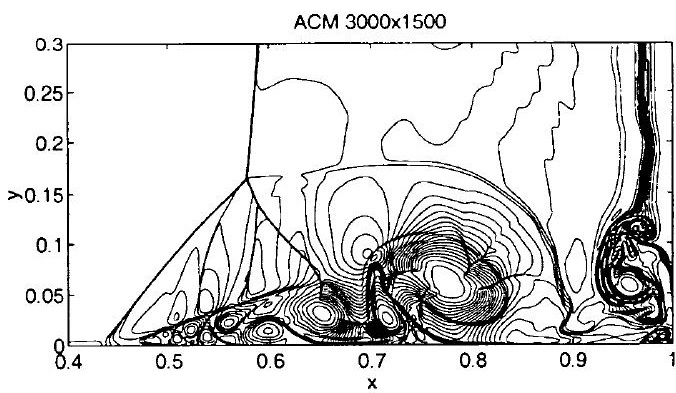

(f) ACM66-RK4, $3000 \times 1500$ grid

Figure 6.12. Comparison: MUSCL-RK2, WAV66-RK4, WAV66-RK4 and WENO5-RK4 for $R e=1000$. Density contours using $1000 \times 500$ and $3000 \times 1500$ grids. 


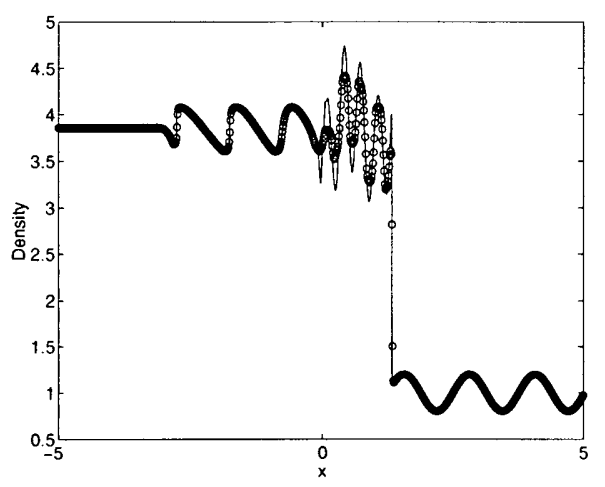

(a) MUSCL-RK2, 800 grid

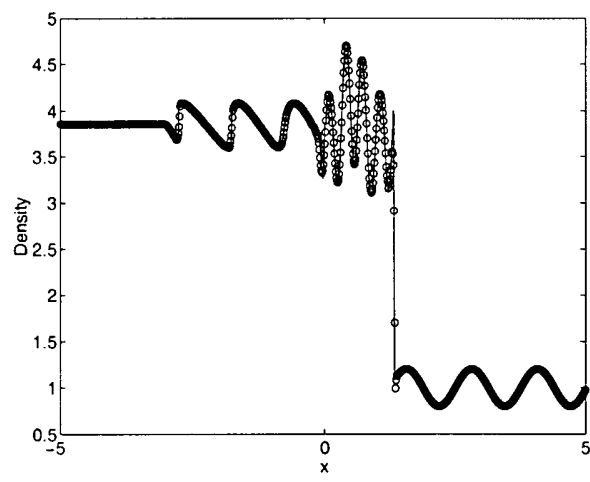

(c) WAV66-RK4-D8, 800 grid

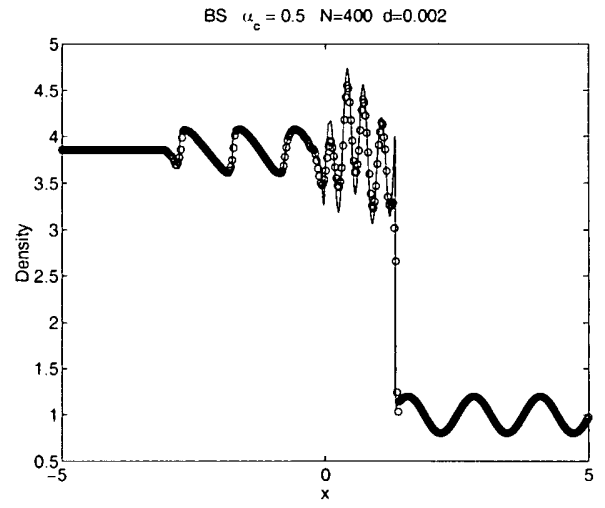

(b) WAV66-RK4-D8, 400 grid
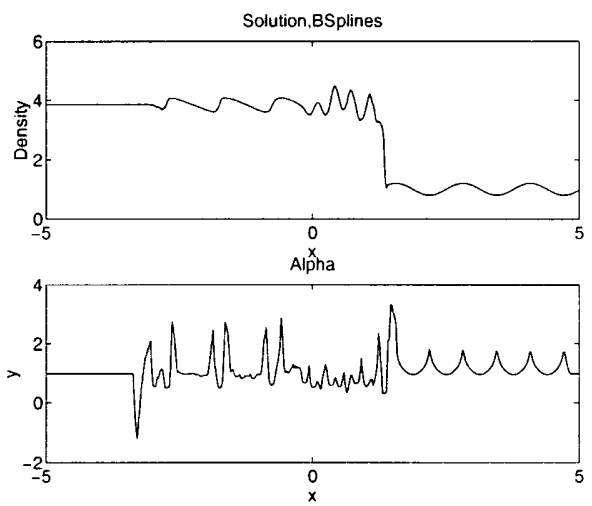

(d) Density \& Lipschitz Exponent Distributions, WAV66-RK4-D8, 400 grid

Figure 6.13. Comparison and Lipschitz exponent distribution of WAV66-RK4-D8, secondorder B-spline wavelet.

turned off (i.e., only the nonlinear filter remains - WAV66-RK4), the computation is not very stable unless a finer grid and smaller time step is used. Turning on the entropy splitting away from the shocks helps to reduce the amount of the eighth-order dissipation coefficient [65].

\section{Concluding Remarks}

An integrated approach for the control of the numerical-dissipation/filter in high order schemes for numerical simulations of multiscale complex flow 
problems is presented. The approach is an attempt to further improve nonlinear stability, accuracy and efficiency of long-time numerical integration of complex shock/turbulence/acoustics interactions and numerical combustion. The required type and amount of numerical-dissipation/filter for these flow problems are not only physical problem dependent, but also vary from one flow region to another. Among other design criteria, the key idea consists of automatic detection of different flow features as distinct sensors to signal the appropriate type and amount of numerical-dissipation/filter for non-dissipative high order schemes. These scheme-independent sensors are capable of distinguishing shocks/shears, turbulent fluctuations and spurious high-frequency oscillations. In addition, these sensors are readily available as an improvement over existing grid adaptation indicators. The same shock/shear detector that is designed to switch on the shock/shear numerical dissipation can be used to switch off the entropy splitting form of the inviscid flux derivative in the vicinity the discontinuous regions to further improve nonlinear stability and minimize the use of numerical dissipation. The rest of the sensors in conjunction with the local flow speed and Reynolds number can also be used to adaptively determine the appropriate entropy splitting parameter for each flow type/region. The minimization of employing very fine grids to overcome the production of spurious numerical solutions and/or instability due to under-resolved grids is also illustrated $[79,17]$. Test examples shown are very encouraging. Full implementation of the approach for practical problems is in progress.

\section{Acknowledgment}

The authors wish to thank their guest author Paul Fischer for contributing to Section 5.5. They also wish to thank their collaborators Neil Sandham, Jahed Djomehri, Marcel Vinokur and Bernhard Müller for contributing to their earlier work. Special thanks to Marcel Vinokur and Tom Coakley for their critical review of the manuscript. The first author would like to acknowledge the support from the Swedish Natural Science Research Council (NFR).

\section{Appendix}

We here give boundary modified numerical dissipation operators, such that the semi bounded property is satisfied for the total operator. The operators are derived by splitting the periodic operator into one boundary part and one interior part,

$$
\left(D_{+} D_{-}\right)_{p e r}=\left(D_{+} D_{-}\right)_{b}+\left(D_{+} D_{-}\right)_{I} .
$$

The interior operator $\left(D_{+} D_{-}\right)_{I}$ has zeros in all rows where the centered operator can not be applied. We define the boundary modified operator as

$$
\left(D_{+} D_{-}\right)^{q}=\left(D_{+} D_{-}\right)_{p e r}^{p}\left(D_{+} D_{-}\right)_{I}^{p}
$$


if $q=2 p$ is even. The zero terms on the boundary of the interior operator, will destroy the periodic terms, so that the boundary modified operator does not have any periodic wrap around terms. If $q=2 p+1$ is odd, we define

$$
\left(D_{+} D_{-}\right)^{q}=\left(D_{+} D_{-}\right)_{p e r}^{p}\left(D_{+}\right)_{p e r}\left(D_{-}\right)_{I}\left(D_{+} D_{-}\right)_{I}^{p} .
$$

The semi boundedness then follows by applying the summation by parts property for the periodic operator, or for the case of $q$ even,

$$
\begin{aligned}
\left(u_{j},\left(D_{+} D_{-}\right)^{q} u_{j}\right)_{h} & =-\left(\left(D_{+} D_{-}\right)_{p e r}^{p} u_{j},\left(D_{+} D_{-}\right)_{I}^{p} u_{j}\right)_{h}= \\
& -\left(\left(D_{+} D_{-}\right)_{I}^{p} u_{j},\left(D_{+} D_{-}\right)_{I}^{p} u_{j}\right)_{h} \leq 0
\end{aligned}
$$

where the last equality follows since the interior operator in the scalar product kills all boundary terms in the periodic operator. The case with odd $q$ can be treated similarly.

The order of the operator is reduced on the boundary. To have correct scaling, as numerical dissipation, the $2 q$ th derivative should be multiplied by $h^{2 q-1}$, thus affecting the accuracy up to order $2 q-1$. However, the boundary terms will be reduced to $q$ th derivatives, and thus have order $q-1$ on the boundary. For example, the fourth order operator will be third order in the interior, and first order on the boundary. The sixth order operator will be fifth order in the interior, and second order on the boundary, etc. We present below examples for orders 4,6 , and 8 .

\section{Fourth derivative}

$$
\left(\begin{array}{cccccccccc}
1 & -2 & 1 & 0 & 0 & 0 & 0 & 0 & 0 & 0 \\
-2 & 5 & -4 & 1 & 0 & 0 & 0 & 0 & 0 & 0 \\
1 & -4 & 6 & -4 & 1 & 0 & 0 & 0 & 0 & 0 \\
0 & 1 & -4 & 6 & -4 & 1 & 0 & 0 & 0 & 0 \\
0 & 0 & 1 & -4 & 6 & -4 & 1 & 0 & 0 & 0 \\
& \ldots & & & & & & & &
\end{array}\right)
$$

\section{Sixth derivative}

$$
\left(\begin{array}{cccccccccc}
-1 & 3 & -3 & 1 & 0 & 0 & 0 & 0 & 0 & 0 \\
3 & -10 & 12 & -6 & 1 & 0 & 0 & 0 & 0 & 0 \\
-3 & 12 & -19 & 15 & -6 & 1 & 0 & 0 & 0 & 0 \\
1 & -6 & 15 & -20 & 15 & -6 & 1 & 0 & 0 & 0 \\
0 & 1 & -6 & 15 & -20 & 15 & -6 & 1 & 0 & 0 \\
0 & 0 & 1 & -6 & 15 & -20 & 15 & -6 & 1 & 0 \\
& \ldots & & & & & & & &
\end{array}\right)
$$


Eighth derivative

$$
\left(\begin{array}{cccccccccc}
1 & -4 & 6 & -4 & 1 & 0 & 0 & 0 & 0 & 0 \\
-4 & 17 & -28 & 22 & -8 & 1 & 0 & 0 & 0 & 0 \\
6 & -28 & 53 & -52 & 28 & -8 & 1 & 0 & 0 & 0 \\
-4 & 22 & -52 & 69 & -56 & 28 & -8 & 1 & 0 & 0 \\
1 & -8 & 28 & -56 & 70 & -56 & 28 & -8 & 1 & 0 \\
0 & 1 & -8 & 28 & -56 & 70 & -56 & 28 & -8 & 1 \\
& \ldots & & & & & & & &
\end{array}\right)
$$





\section{References}

[1] M. Alam and N.D. Sandham, DNS of Transition Near the Leading Edge of an Aerofoil. Proc Direct and Large Eddy Simulation 4, B.J.Geurts, ed, to appear Kluwer (2001).

[2] Alpert, P., Implicit Filtering in Conjunction with Explicit Filtering, J. Comput. Phys. 44, 212-219 (1981).

[3] M. Ashworth, D.R. Emerson, N.D. Sandham, Y.F. Yao, and Q. Li, Parallel DNS Using a Compressible Turbulent Channel Flow Benchmark. Proc. ECCOMAS CFD Conference, Swansea, Wales, 4-7 Sept. 2001.

[4] A. Arakawa, Computational Design for Long-Term Numerical Integration of the Equations of Fluid Motion: Two-Dimensional Incompressible Flow. Part I, J. Comput. Phys., 1, pp. 119-143 (1966).

[5] G. A. Blaisdell, Numerical Simulation of Compressible Homogeneous Turbulence, $\mathrm{PhD}$ Thesis, Stanford University, 1991.

[6] Brown, D.L. and Minion, M.L., Performance of Under-resolved Two-Dimensional Incompressible Flow Simulations, J. Comput. Phys. 122, 165-183 (1995).

[7] Brown, D.L., Margolin, L., Sharp, D. and A. White, A., Predictability of Complex Phenomena, A White Paper, Los Alamos National Laboratory reprint (1997).

[8] M.H. Carpenter, D. Gottlieb, S. Abarbanel S. and W.-S. Don, The Theoretical Accuracy of Runge-Kutta Time Discretizations for Initial Value Problem: A Study of the Boundary Error, SIAM J. Sci. Comput. 16, 1241-1252 (1995).

[9] M.H. Carpenter, J. Nordstrom and D. Gottlieb, A Stable and Conservative Interface Treatment of Arbitrary Spatial Accuracy, ICASE Report 98-12, 1998.

[10] M.H. Carpenter, D. Gottlieb and S. Abarbanel, Time-Stable Boundary Conditions for Finite-Difference Schemes Solving Hyperbolic Systems: Methodology and Application to High-Order Compact Schemes, J. Comput. Phys., 111, 220-236 (1994).

[11] G.N. Coleman, J. Kim, J. and R. Moser, A Numerical Study of Turbulent Supersonic Isothermal-Wall Channel Flow, J. Fluid Mech. 305, pp. 159-183 (1995).

[12] V. Daru and C. Tenaud, Evaluation of TVD High Resolution Schemes for Unsteady Viscous Shocked Flows, Computers Fluids, 30 89-113 (2000).

[13] I. Daubechies, Ten Lectures on Wavelets, CBMS-NSF Regional Conference Series in Applied Mathematics, No 61, SIAM, 1992. 
[14] B. Engquist and B. Sjögreen, High Order Shock Capturing Methods, CFD Reviews, (Hafez \& Oshima, Eds.), John Wiley, New York, 210-233 (1995).

[15] M. Farge, Wavelet Transforms and their Applications to Turbulence, Ann. Rev. of Fluid Mech., 24, 395 (1992).

[16] W. J. Feiereisen, W. C. Reynolds, and J. H. Ferziger, Numerical Simulation of a Compressible Homogeneous, Turbulent Shear Flow, Report No. TF-13 (1981), Thermosciences Division, Department of Mechanical Engineering, Stanford University.

[17] P.F. Fischer and J.S. Mullen, Filter-Based Stabilization of Spectral Element Methods, Argonne National Lab. Report, August 4, 1999.

[18] D.V. Gaitone and M.R. Visbal, Further Development of a Navier-Stokes Solution Procedure Based on Higher-Order Formulas, AIAA Paper 99-0557, Reno, NV, 1999.

[19] P. R. Garabedian, Partial Differential Equations, John Wiley \& Sons, 1964.

[20] M. Gerritsen and P. Olsson, Designing an Efficient Solution Strategy for Fluid Flows I. J. Comput. Phys., 129, pp. 245-262 (1996).

[21] J. Glimm and D. Sharp, Prediction and the Quantification of Uncertainty, Los Alamos National Laboratory LAUR-98-3391 (1998).

[22] D. Gottlieb and J.S. Hesthaven, Spectral Methods for Hyperbolic Problems, J. Comput. Applied Math., 128, 83-131 (2001).

[23] P.M. Gresho, D.K. Gartling, J.R. Torczynski, K.A. Cliffe, K.H. Winters, T.J. Garrett, A. Spence and J.W. Goodrich, Is the Steady Viscous Incompressible Two-Dimensional Flow Over a Backward-facing Step at Re $=800$ Stable?, Intern. J. Numer. Meth. Fluids 17 501-541 (1993).

[24] B. Gustafsson, H.-O. Kreiss and J. Oliger, J., Time Dependent Problems and Difference Methods, John Wiley \& Sons, New York (1995).

[25] A. Harten, On the Symmetric Form of Systems for Conservation Laws with Entropy, J. Comput. Phys. 49, 151-164 (1983).

[26] R. D. Henderson, Adaptive Spectral Element Methods for Turbulence and Transition, in High-Order Methods for Computational Physics, T. J. Barth and H. Deconinck, editors. Springer 1999.

[27] C.W. Hirt, Heuristic Stability Theory for Finite-Difference Equations, J. Comput. Phys., 2, pp. 339-355 (1968).

[28] Huang, P. G., G.N. Coleman, and P. Bradshaw, Compressible Turbulent Channel Flows: DNS Results and Modeling. J. Fluid Mech. 305, 185-218 (1995).

[29] M. Johansson, Loss of High Order Spatial Accuracy due to Boundary Error Caused by Runge-Kutta Time Integration, Technical report 2000-013, Dept. Info. Tech., Uppsala University, May 2000.

[30] H.-O. Kreiss and J. Oliger, Comparison of accurate methods for the integration of hyperbolic equations, Tellus, 24, pp. 199-215 (1972). 
[31] H.-O. Kreiss, Numerical Methods for Solving Time-Dependent Problems for Partial Differential Equations, Les Presses de l'Université de Montréal, 1978.

[32] J. Kim, P. Moin and R.D. Moser, Turbulence Statistics in fully-Developed Channel Flow at Low Reynolds Number. J. Fluid Mech. 177, 133-166 (1987).

[33] Lafon, A. and Yee, H.C., Dynamical Approach Study of Spurious Steady-State Numerical Solutions for Nonlinear Differential Equations, Part III: The Effects of Nonlinear Source Terms in Reaction-Convection Equations, Comp.. Fluid Dyn. 6 1-36 (1996).

[34] Lafon, A. and Yee, H.C., Dynamical Approach Study of Spurious Steady-State Numerical Solutions of Nonlinear Differential Equations, Part IV: Stability vs. Numerical Treatment of Nonlinear Source Terms, Comput. Fluid Dyn. 6 89-123 (1996).

[35] A.A. Lawal, and N.D. Sandham, Direct Simulation of Transonic Flow Over a Bump. Proc Direct and Large Eddy Simulation 4, B.J.Geurts, ed, to appear Kluwer (2001).

[36] LeVeque, R.J. and Yee, H.C., A Study of Numerical Methods for Hyperbolic Conservation Laws with Stiff Source Terms, J. Comput. Phys. 86 187-210 (1990).

[37] Lele. S.A., Compact Finite Difference Schemes with Spectral-Like Resolution, J. Comput. Phys., 103, 16-42 (1992).

[38] Q. Li, Direct Numerical Simulation of Compressible Turbulent Channel Flow. PhD Thesis, University of Southampton, in preparation (2001).

[39] R.W. MacCormack, Numerical Solution of the Interaction of a Shock Wave with a Laminar Boundary Layer, Proceedings of the 2nd Intern. Conf. on Num. Meths. in Fluid Dynamics, pp. 151-163 (1971).

[40] Y. Maday and A. T. Patera, Spectral element methods for the Navier-Stokes equations, State of the Art Surveys in Computational Mechanics, A. K. Noor, ed., ASME, New York, pp. 71-143 (1989).

[41] S.G. Mallat, $A$ Wavelet Tour of Signal Processing, Second Edition, Academic Press, San Diego (1999).

[42] S.G. Mallat, Multifrequency channel Decompositions of Images and Wavelet Models, IEEE Trans. Acoust. Speech Signal Process., 37, 2091 (1989).

[43] S.G. Mallat, Multiresolution Approximations and Wavelet Orthonormal Bases of $L^{2}(R)$, Trans. Amer. Math. Soc., 315, 69 (1989).

[44] S.G. Mallat, A Theory for Multiresolution Signal Decomposition: The Wavelet Representation, IEEE Trans. Patt. Anal. Mach. Intell., 11, 674 (1989).

[45] S.G. Mallat and W. L. Hwang, Singularity Detection and Processing with Wavelets, IEEE Transactions on Information Theory, 38, 617 (1992).

[46] S.G. Mallat and S. Zhong, Characterization of Signals from Multiscale Edges, IEEE Transactions on Pattern Analysis and Machine Intelligence, 14 710-732 (1992).

[47] N. N. Mansour, J. H. Ferziger, and W. C. Reynolds, Large-Eddy Simulation of a Turbulent Mixing Layer, Report TF-11, Thermosciences Division, Stanford University, (1978). 
[48] K. Mattsson, Imposing Boundary Conditions with the Injection, the Projection and the Simultaneous Approximation Term Methods, Proceedings of the First International Conference on CFD, July 10-14, 2000, Kyoto, Japan.

[49] M.L. Minion, and D.L. Brown, Performance of Under-resolved Two-Dimensional Incompressible Flow Simulations II, J. Comput. Phys. 138, 734-765 (1997).

[50] B. Müller and H.C. Yee, Entropy Splitting for High Order Numerical Simulation of Vortex Sound at Low Mach Numbers, Proceedings of the 5th Internat. Conf. on Spectral and High Order Methods, Uppsala, Sweden, June 11-15, 2001.

[51] J. Nordström and M.H. Carpenter, Boundary and Interface Conditions for High-Order Finite-Difference Methods Applied to the Euler and Navier-Stokes Equations, J. Comput. Phys., 148, 621-645 (1999).

[52] W.L. Oberkampf, K.V. Diegert, K.F. Alvin, and B.M. Rutherford, Variability, Uncertainty and Error in Computational Simulation, ASME Proceedings of the 7th. AIAA/ASME Joint Thermophysics and Heat Transfer Conference, HTD-Vol. 357-2 (1998).

[53] P. Olsson, Summation by Parts, Projections, and Stability. I, Math. Comp. 64 (1995), pp. 1035-1065.

[54] P. Olsson, Summation by Parts, Projections, and Stability. II, Math. Comp. 64, pp. 14731493 (1995).

[55] P. Olsson, Summation by Parts, Projections, and Stability. III, RIACS Technical Report 95.06 (1995).

[56] P. Olsson and J. Oliger, Energy and Maximum Norm Estimates for Nonlinear Conservation Laws, RIACS Technical Report 94.01, (1994).

[57] V. Perrier, T. Philipovitch, and C. Basdevant, Wavelet Spectra Compared to Fourier Spectra, Publication of ENS, Paris, 1999.

[58] R. D. Richtmyer and K. W. Morton, Difference Methods for Initial-Value Problems, 2nd ed., John Wiley \& Sons, 1967.

[59] N.D. Sandham and R.J.A. Howard, Direct Simulation of Turbulence Using Massively Parallel Computers. Parallel Computational Fluid Dynamics, Ed. D.R.Emerson et al., Elsevier (1998).

[60] Sandham, N.D. and Yee, H.C., Entropy Splitting for High Order Numerical Simulation of Compressible Turbulence, RIACS Technical Report 00.10, June, 2000, Proceedings of the 1st Intern. Conf. on CFD, July 10-14, 2000, Kyoto, Japan.

[61] N. D. Sandham, Q. Li and H.C. Yee, Entropy Splitting for High Order Numerical Simulation of Compressible Turbulence, Revised version of RIACS Technical Report 00.10, June 2000, NASA Ames Research Center; Proceedings of the First International Conference on CFD, July 10-14, 2000, Kyoto, Japan.

[62] B. Sjögreen, Numerical Experiments with the Multiresolution Scheme for the Compressible Euler Equations, J. Comput. Phys., 117, 251 (1995). 
[63] B. Sjögreen and H. C. Yee, Multiresolution Wavelet Based Adaptive Numerical Dissipation Control for Shock-Turbulence Computations, RIACS Report 01.01, NASA Ames research center (Oct 2000).

[64] B. Sjögreen and H. C. Yee, Grid Convergence of High Order Methods for Multiscale Complex Unsteady Viscous Compressible Flows, RIACS Report 01.06, April, 2001, NASA Ames research center; AIAA 2001-2599, Proceedings of the 15th AIAA CFD Conference, June 11-14, 2001, Anaheim, CA.

[65] B. Sjögreen and H.C. Yee, On Entropy Splitting, Linear and Nonlinear Numerical Dissipations and Long-Time Integrations, Proceedings of the 5th Internat. Conf. on Spectral and High Order Methods, Uppsala, Sweden, June 11-15, 2001.

[66] B. Sjögreen and H. C. Yee, Low Dissipative High Order Numerical Simulations of Supersonic Reactive Flows, RIACS Report 01-017, NASA Ames Research Center (May 2001); Proceedings of the ECCOMAS Computational Fluid Dynamics Conference 2001, Swansea, Wales, UK, September 4-7, 2001.

[67] B. Sjögreen, High Order Centered Difference Methods for the Compressible Navier-Stokes Equations, J. Comput. Phys., 117, pp. 67-78 (1995).

[68] Special Section of the AIAA Journal, Credible Computational Fluid Dynamics Simulation, AIAA J. 36, 665-759 (1998).

[69] Strand, B., High-Order Difference Approximations for Hyperbolic Initial Boundary Value Problems, PhD thesis, Uppsala University, Department of Scientific Computing, 1996.

[70] B. Strand, Summation by Parts for Finite Difference Approximations for $d / d x$, J. Comput. Phys. 110, pp. 47-67 (1994).

[71] G. Strang, Wavelet Transforms versus Fourier Transforms, Bull. Amer. Math. Soc. (N.S.), 28, 288 (1993).

[72] G. Strang, Wavelet and Dilation Equations: A Brief Introduction, SIAM Rev., 31, 614 (1989).

[73] E. Tadmor, Skew-Selfadjoint Form for Systems of Conservation Laws, J. Math. Anal. Appl., 103, pp. 428-442 (1984).

[74] J.R. Torczynski, A Grid Refinement Study of Two-Dimensional Transient Flow Over a Backward-facing Step Using a Spectral-Element Method, FED-Vol. 149, Separated Flows, ASME, J.C. Dutton and L.P. Purtell, Eds. (1993).

[75] T.G. Trucano, Prediction and Uncertainty in Computational Modeling of Complex Phenomena: A Whitepaper, Sandia Report, SAND98-2776 (1998).

[76] R. Vichnevetsky, Numerical Filtering for Partial Differencing Equations, Numerical Applications Memorandum, Rutgers University, NAM 156 (1974).

[77] M. Vinokur and H.C. Yee, Extension of Efficient Low Dissipative High Order Schemes for 3-D Curvilinear Moving Grids, NASA TM 209598, June 2000.

[78] Yee, H.C., Sweby, P.K. and Griffiths, D.F., Dynamical Approach Study of Spurious SteadyState Numerical Solutions for Nonlinear Differential Equations, Part I: The Dynamics of 
Time Discretizations and Its Implications for Algorithm Development in Computational Fluid Dynamics, NASA TM-102820, April 1990; J. Comput. Phys. 97 249-310 (1991).

[79] H.C. Yee, P.K. Sweby, Dynamics of Numerics Spurious Behaviors in CFD Computations, 7th ISCFD Conference, Sept. 15-19, 1997, Beijing, China, RIACS Technical Report 97.06, June (1997).

[80] H.C. Yee and P.K. Sweby, Some Aspects of Numerical Uncertainties in Time Marching to Steady-State Computations, AIAA-96-2052, 27th AIAA Fluid Dynamics Conference, June 18-20, 1996, New Orleans, LA., AIAA J., 36 712-724 (1998).

[81] H.C. Yee, J.R. Torczynski, S.A. Morton, M.R. Visbal and P.K. Sweby, On Spurious Behavior of CFD Simulations, AIAA 97-1869, Proceedings of the 13th AIAA Computational Fluid Dynamics Conference, June 29 - July 2, 1997, Snowmass, CO.; also International J. Num. Meth. Fluids, 30, 675-711 (1999).

[82] H. C. Yee, N. D. Sandham, and M. J. Djomehri, Low Dissipative High Order ShockCapturing Methods Using Characteristic-Based Filters, J. Comput. Phys., 150 199-238 (1999).

[83] H.C. Yee, M. Vinokur, and M.J. Djomehri, Entropy Splitting and Numerical Dissipation, NASA Technical Memorandum 208793, August, 1999, NASA Ames Research Center; J. Comput. Phys., 162, 33 (2000).

[84] H.C. Yee and B. Sjögreen, Adaptive Numerical-Dissipation/Filter Controls for High Order Numerical Methods, Proceedings of the 3rd International Conference on DNS/LES, Arlington, Texas, August 4-9, 2001.

[85] S.T. Zalesak, High Order "ZIP" Differencing of Convective Terms, J. Comput. Phys., 40 (1981), pp. 497-508.

[86] T. A. Zang, On the Rotation and Skew-Symmetric Forms for Incompressible Flow Simulations, Appl. Numer. Math., 7 (1991), pp. 27-40. 